\title{
Kernel Contraction and Base Dependence
}

Mehrdad Oveisi

Department of Computer Science, University of British Columbia Vancouver, BC, V6T 1Z4, Canada

James P. Delgrande

School of Computing Science, Simon Fraser University

Burnaby, BC, V5A 1S6, Canada

Francis Jeffry Pelletier

Department of Philosophy, University of Alberta

Edmonton, AB, T6G 2E7, Canada

Fred Popowich

School of Computing Science, Simon Fraser University

Burnaby, BC, V5A 1S6, Canada
MOVEISI@CS.UBC.CA

JIM@CS.SFU.CA

FRANCISP@UALBERTA.CA

POPOWICH@CS.SFU.CA

\begin{abstract}
The AGM paradigm of belief change studies the dynamics of belief states in light of new information. Finding, or even approximating, those beliefs that are dependent on or relevant to a change is valuable because, for example, it can narrow the set of beliefs considered during belief change operations. A strong intuition in this area is captured by Gärdenfors's preservation criterion (GPC), which suggests that formulas independent of a belief change should remain intact. GPC thus allows one to build dependence relations that are linked with belief change. Such dependence relations can in turn be used as a theoretical benchmark against which to evaluate other approximate dependence or relevance relations. Fariñas and Herzig axiomatize a dependence relation with respect to a belief set, and, based on GPC, they characterize the correspondence between AGM contraction functions and dependence relations. In this paper, we introduce base dependence as a relation between formulas with respect to a belief base, and prove a more general characterization that shows the correspondence between kernel contraction and base dependence. At this level of generalization, different types of base dependence emerge, which we show to be a result of possible redundancy in the belief base. We further show that one of these relations that emerge, strong base dependence, is parallel to saturated kernel contraction. We then prove that our latter characterization is a reversible generalization of Fariñas and Herzig's characterization. That is, in the special case when the underlying belief base is deductively closed (i.e., it is a belief set), strong base dependence reduces to dependence, and so do their respective characterizations. Finally, an intriguing feature of Fariñas and Herzig's formalism is that it meets other criteria for dependence, namely, Keynes's conjunction criterion for dependence (CCD) and Gärdenfors's conjunction criterion for independence $(\mathrm{CCl})$. We prove that our base dependence formalism also meets these criteria. Even more interestingly, we offer a more specific criterion that implies both $\mathrm{CCD}$ and $\mathrm{CCl}$, and show our base dependence formalism also meets this new criterion.
\end{abstract}

\section{Introduction: Belief Change}

Research into belief change provides a formal means for incorporating new and changing information. Alchourrón, Gärdenfors and Makinson (1985) provide the AGM paradigm of belief change 
that idealizes a belief state as a belief set $K$ : a set of logical formulas that is closed under logical consequence. One example of a belief change operation on $K$ is contraction which retracts a formula $\alpha$ and whatever other formulas of $K$ that would be necessary to ensure $\alpha$ is not implied by the remaining formulas.

In an important variant of the original AGM approach, a belief base (usually denoted by $B$ ) is used instead of a belief set. Belief bases are not necessarily deductively closed, and they are usually finite. Thus, they are more suitable to be represented in finite machines and arguably better as models of cognitive agents. Also, many authors have argued that, compared to belief sets, belief bases are more expressive (Hansson, 2003), and they are more tolerant of inconsistency (Hansson \& Wassermann, 2002). Therefore, belief bases may be more useful in practice than belief sets.

\subsection{Belief Change and Dependence}

During a belief change operation, beliefs independent of a change should remain intact. Gärdenfors states this intuition in the following preservation criterion (Gärdenfors, 1990):

"If a belief state is revised by a sentence $\mathrm{A}$, then all sentences in $\mathrm{K}$ that are independent of the validity of A should be retained in the $(\mathrm{GPC})$ revised state of belief."

In Belief Change and Dependence, Fariñas del Cerro and Herzig (1996) ${ }^{1}$ attempt to ground the intuition stated in GPC, and aim both to "give a formal account of the notion of dependence, and to employ it in belief change." FH's work is particularly interesting and unique in the sense that it fits the original AGM model of belief change. This deep integration into the AGM model sets apart their work from other works on relevance or dependence in the context of belief change.

\subsection{Belief Change and Base Dependence}

A natural next step is to find a similar connection between dependence and belief base contraction. We call such a dependence (or relevance) relation base dependence (or base relevance). In this paper, we provide an axiomatization of base dependence, and establish its relation to belief base contraction. Indeed, in both studies based on GPC (FH's and ours), GPC allows us to build dependence relations that are theoretically linked with belief change. Such dependence relations can in turn be used, for example, as a theoretical benchmark against which to evaluate other approximate dependence or relevance relations. When generalizing the concept of dependence for belief bases, different types of base dependence emerge, namely, strong base dependence and weak base dependence ( $\$ 3.5)$. We show that weak base dependence is a result of redundancy in the belief base (§3.5.3). This is another result from our study that can be used as a theoretical benchmark in other studies. Additionally, the fact that weak base dependence captures redundancy may be exploited for various purposes. For example, one may use weak base dependence to distinguish between the redundant and the informative formulas of a belief base.

Moreover, strong base dependence interestingly turns out to be a reversible generalization of FH's dependence ( $(5)$. That is, in the special case that a belief base is deductively closed (i.e., it is a belief set), the strong base dependence relation reduces to FH's original dependence relation.

1. Because of our frequent citation of this particular article, we will use 'FH' to refer to it in the rest of this article. 


\subsection{Composite Dependence}

One interesting aspect of FH's work is that some of the axioms that they use to capture the concept of dependence come from intuitions that have appeaared in the literature in other contexts. For example, Keynes (1921) holds that there is an intuitive relationship between relevance (dependence) and logical conjunction that should be valid for any reasonable definition of relevance. Calling Keynes's view the Conjunction Criterion for Dependence, CCD, FH formulate it as follows:

If $\delta$ depends on $\alpha$ and $\delta$ depends on $\beta$ then $\delta$ depends on $\alpha \wedge \beta$.

$(C C D)$

Gärdenfors (1978) also puts forward another principle that he believes should hold for relevance/dependence relations, the Conjunction Criterion for Independence, $\mathrm{CCl}$.

If $\delta$ is independent of $\alpha$ and $\delta$ is independent of $\beta$ then $\delta$ is independent of $\alpha \wedge \beta$.

This principle maintains its intuitive appeal in its contrapositive form:

If $\delta$ depends on $\alpha \wedge \beta$ then $\delta$ depends on $\alpha$ or $\delta$ depends on $\beta$.

Our formalism will preserve both CCD and CCl. We also offer a new and more specific criterion for dependence, which we call the Conjunction Criterion of Dependence Factoring, CCDF. We show that CCDF implies both CCD and $\mathrm{CCl}$, and that our base dependence formalism meets the three criteria: CCDF and therefore CCD and $\mathrm{CCl}$.

\subsection{Contributions}

The contributions in this paper are as follows. We study the base dependence relation ( $\$ 3)$, and we offer an axiomatization of base dependence for belief base formulas ( $\$ 3.3)$. We then provide characterization theorems relating base dependence to belief base contraction ( $\S 3.4$ ), and, more specifically, we offer a correspondence between kernel contraction and base dependence ( $\$ 4.1)$.

We further show that base dependence has two mutually exclusive subclasses: strong base dependence and weak base dependence. We prove that this differentiation of base dependence types is a result of possible redundancy in a belief base ( $(3.5)$. We show that strong base dependence is a reversible generalization of FH's dependence relation, and it corresponds to saturated kernel contraction $(\S 4.2, \S 5)$. We also show that although it generalizes the dependence relation, strong base dependence nevertheless preserves some of the most interesting properties of dependence, particularly, Keynes's CCD, and Gärdenfors's CCI (§4.3). Furthermore, we put forward a more specific conjunction criterion of dependence, CCDF, that implies both CCD and CCI (§4.3). We show that this new criterion is also met by strong base dependence $(\S 5)$. All the proofs are presented in Appendix A. This article is an extension and elaboration of the works published by the present authors (Oveisi, 2013; Oveisi, Delgrande, Pelletier, \& Popowich, 2014; Oveisi, Delgrande, Popowich, \& Pelletier, 2015).

\section{Background}

In this section we give the formal background to belief change and various operations that are prevalent in the literature, setting the stage for our own contributions. 


\subsection{Formal Preliminaries}

We assume $\mathcal{L}$ to be a propositional language defined on a finite set of propositional variables or atoms $\mathcal{V}$ with Boolean operators negation $\neg$, conjunction $\wedge$, disjunction $\vee$, and implication $\rightarrow$. For metavariables over sentences in $\mathcal{L}$, we use Greek letters $\alpha, \beta, \delta$, etc. We introduce the sentential constants $T$ and $\perp$ for convenience, representing truth and falsity respectively. $B \vdash \alpha$ expresses that $\alpha$ is a logical consequence of a set of formulas $B$. Cn is a consequence operator, defined as $\operatorname{Cn}(B)=\{\alpha \mid B \vdash \alpha\}$. It is a total function taking sets of formulas to sets of formulas. Cn is a Tarskian (1956) consequence operator satisfying:

$$
\begin{aligned}
& B \subseteq \operatorname{Cn}(B) \\
& \text { If } A \subseteq B \text { then } \operatorname{Cn}(A) \subseteq \operatorname{Cn}(B) \\
& \operatorname{Cn}(B)=\operatorname{Cn}(\operatorname{Cn}(B))
\end{aligned}
$$$$
\text { (inclusion) }
$$

(iteration)

Furthermore, the Cn operator is assumed here to satisfy the following standard properties:

If $\alpha$ can be derived from $B$ by classical truth-functional logic then $\alpha \in \operatorname{Cn}(B)$

$\beta \in \operatorname{Cn}(B \cup\{\alpha\})$ iff $(\alpha \rightarrow \beta) \in \operatorname{Cn}(B)$

(supraclassicality)

If $\alpha \in \operatorname{Cn}(B)$ then $\alpha \in \operatorname{Cn}\left(B^{\prime}\right)$ for some finite subset $B^{\prime} \subseteq B$.

(deduction)

(compactness)

We generally use $K$ only for logically closed sets, $K=\operatorname{Cn}(K)$, while $B$ may be used to refer to either closed or non-closed sets.

\subsection{Belief Change}

The AGM framework has been the most influential work to formalize the dynamics of belief states of an intelligent agent. This framework was developed by Carlos Alchourrón, Peter Gärdenfors, and David Makinson (Gärdenfors, 1984; Alchourrón, Gärdenfors \& Makinson, 1985; Gärdenfors, 1988; Gärdenfors \& Makinson, 1988), thus the acronym AGM. The framework uses sentences from a language $\mathcal{L}$ to represent beliefs about the world or a situation. The belief state of an idealized intelligent agent, who knows all the consequences of its beliefs, is then represented using a formal theory, which as we mentioned earlier is called a belief set - a set of beliefs alongside all its logical consequences, $K=\mathrm{Cn}(K)$. The logical closure property of belief sets is a simplifying assumption that is removed in some important extensions of AGM.

Let us assume that there is an intelligent agent possessing a consistent set of beliefs $K$ about some domain. Now, what happens if some credible new information $\alpha$ about the domain becomes available? How should the agent revise its old beliefs to incorporate the new information, and end up with a new, consistent body of beliefs? Reconsidering old beliefs is not always easy, nor necessarily wise, thus the change should be as little as possible, and only as much as required to avoid any conflict between the new information and the old.

A simple possibility that comes to mind is to add the new information $\alpha$ to $K$ along with any implications this addition may have. This operation is called expansion, denoted by + , which can be defined as $K+\alpha=\operatorname{Cn}(K \cup\{\alpha\})$.

It is apparent that expansion can bring about inconsistent results when $\alpha$ is in conflict with the current belief set $K$. In this case, the agent first needs to remove some of its old beliefs, albeit as 
few as possible, to resolve any conflicts with the new information. Such an operation that guarantees consistent incorporation of the new belief $\alpha$ is called revision $*$, and the resulting belief set is denoted by $K * \alpha$. Another belief change operation is called contraction $\div$ which allows an agent to give up on some of its existing beliefs $K$ such that the remaining beliefs and their consequences, denoted by $K \div \alpha$, do not include a given belief $\alpha$. Indeed, revision and contraction are interconnected and each one can be defined using the other. In particular, revision can be defined in terms of contraction using the Levi Identity:

$K * \alpha=(K \div \neg \alpha)+\alpha$

As such, here we only consider contraction, which is further discussed in the following section.

It is noteworthy that, in a given situation, different intelligent agents could change their beliefs differently. In general, it is not possible to uniquely define the belief change operators contraction or revision. Instead, the AGM approach introduces some rationality postulates or axioms on belief change operators that distinguish between operators that result in some rational changes to belief sets, as opposed to the ones making invalid or unnecessary changes, which are rationally unacceptable. In other words, because it is not possible to uniquely define rational belief change operators, instead rationality postulates are used to describe such operators. The AGM framework also provides ways to construct belief change operators. Finally, the framework uses axiomatic characterization theorems to show that this description and construction of belief change operators match.

\subsection{Belief Contraction}

Formally, a contraction operator $\div$ maps a belief set $K$ and formula $\alpha$ to a belief set $K \div \alpha$. The following are rationality postulates that the AGM approach uses to describe what constitute belief contraction operators:

$(\div 1) K \div \alpha$ is a belief set

(closure)

$(\div 2) K \div \alpha \subseteq K$

(inclusion)

$(\div 3)$ If $\alpha \notin K$ then $K \div \alpha=K$

(vacuity)

$(\div 4)$ If $\nvdash \alpha$ then $\alpha \notin K \div \alpha$

$(\div 5)$ If $\alpha \in K$ then $K \subseteq(K \div \alpha)+\alpha$

(recovery)

$(\div 6)$ If $\vdash \alpha \leftrightarrow \beta$ then $K \div \alpha=K \div \beta$

(extensionality)

$(\div 7) K \div \alpha \cap K \div \beta \subseteq K \div \alpha \wedge \beta$

(conjunctive overlap)

$(\div 8)$ If $\alpha \notin K \div \alpha \wedge \beta$ then $K \div \alpha \wedge \beta \subseteq K \div \alpha$

(conjunctive inclusion)

The first axiom $(\div 1)$ states the closure property of contraction function - contracting a belief set always results in a belief set. $(\div 2)$ ensures that contraction does not introduce any new formula, and it may only take away some of the existing ones. By $(\div 3)$, if a formula is not already in a belief set, contraction leaves the belief set unchanged; no change should be made when not necessary. $(\div 4)$ guarantees the resulting belief set from a contraction does not contain the contracted formula, given it is not a tautology. In other words, $\alpha \in K \div \alpha$ can happen only for a tautological $\alpha$. By 
$(\div 5)$, the original belief set can be recovered after contraction by re-expanding the result by the original contracted formula. $^{2}(\div 6)$ states that contraction is syntax independent.

Any operator $\div$ on $K$ satisfying postulates $(\div 1)-(\div 6)$ is called a basic AGM contraction function. The supplementary postulates $(\div 7)$ and $(\div 8)$ specify properties of composite belief contraction functions, which involve contraction by a conjunction of sentences. Indeed, the AGM approach also provides a third composite contraction postulate:

Either $K \div \alpha \wedge \beta=K \div \alpha$, or

$$
\begin{aligned}
& K \div \alpha \wedge \beta=K \div \beta, \text { or } \\
& K \div \alpha \wedge \beta=K \div \alpha \cap K \div \beta
\end{aligned}
$$

(conjunctive factoring)

A basic AGM contraction function that satisfies conjunctive factoring, also satisfies both $(\div 7)$ and $(\div 8)$, and vice versa. The relationship between these three axioms is formally stated in the following theorem.

Theorem 1 (Alchourrón, Gärdenfors \& Makinson, 1985). Let $\div$ be an operation on belief set $K$ that satisfies $(\div 1)-(\div 6)$, then both conjunctive overlap $(\div 7)$ and conjunctive inclusion $(\div 8)$ are satisfied if and only if conjunctive factoring is satisfied.

\subsubsection{Belief BASE CONTRACTION}

As mentioned, the original AGM paradigm of belief change studies the dynamics of belief states using belief sets, which are deductively closed, thus infinite in size. Belief bases generalize belief sets, removing the deductive closure requirement. Given a belief base $B$, it is always possible to obtain the corresponding belief set $K$ using the consequence operator: $K=\operatorname{Cn}(B)$. This generalization is advantageous in a few ways. First off, being typically finite in size, belief bases are more suitable to be represented in finite machines and are arguably better as models of cognitive agents. Moreover, there can be many different belief bases whose logical closure is the same belief set. This makes belief bases more expressive compared to belief sets. That is because belief bases can distinguish between explicit, or more basic beliefs, $B$, and implicit beliefs, $\operatorname{Cn}(B) \backslash B$, which depend on the basic beliefs (Hansson, 2003). Another advantage of belief bases is that they allow for the handling of inconsistencies (Hansson \& Wassermann, 2002). For example, let $A=\{p, \neg p, q\}$ and $B=\{p, \neg p, \neg q\}$. Because both $A$ and $B$ are inconsistent, their corresponding belief set is the same: $\operatorname{Cn}(A)=\operatorname{Cn}(B)=\mathcal{L}$. Yet, $A \div p=\{\neg p, q\}$ and $B \div p=\{\neg p, \neg q\}$ are different and so are their closures, $\operatorname{Cn}(A \div p) \neq \operatorname{Cn}(B \div p)$.

The connection between contraction on belief bases and contraction on belief sets is well established. A contraction operator $\div{ }_{1}$ on a belief base $B$ allows one to define a base-generated operation $\div_{2}$ on the belief set $K=\operatorname{Cn}(B)$, such that $K \div_{2} \alpha=\operatorname{Cn}\left(B \div{ }_{1} \alpha\right)$ (Hansson, 1993, 2011).

2. The recovery postulate has turned out to be the most controversial AGM contraction postulate (Makinson, 1987; Fermé, 2001). 
Just as in the case of the AGM operators for belief sets, belief change operators for belief bases are also constrained by a set of postulates, with some of the important ones listed below:

$$
\begin{array}{lr}
B \div \alpha \subseteq B & \begin{array}{r}
\text { (inclusion) } \\
\text { If } \nvdash \alpha \text { then } \alpha \notin \operatorname{Cn}(B \div \alpha)
\end{array} \\
B \cap \operatorname{Cn}(B \div \alpha) \subseteq B \div \alpha & \text { (success) } \\
\text { If } \alpha \in \operatorname{Cn}\left(B^{\prime}\right) \text { iff } \beta \in \operatorname{Cn}\left(B^{\prime}\right) \text { for all } B^{\prime} \subseteq B & \text { (relative closure) } \\
\quad \text { then } B \div \alpha=B \div \beta & \text { (uniformity) } \\
\text { If } \beta \in B \text { and } \beta \notin B \div \alpha \text { then } & \\
\quad \alpha \notin \operatorname{Cn}\left(B^{\prime}\right) \text { and } \alpha \in \operatorname{Cn}\left(B^{\prime} \cup\{\beta\}\right) \text { for some } B \div \alpha \subseteq B^{\prime} \subseteq B & \text { (relevance) } \\
\text { If } \beta \in B \text { and } \beta \notin B \div \alpha \operatorname{then} & \text { (core-retainment) } \\
\quad \alpha \notin \operatorname{Cn}\left(B^{\prime}\right) \text { and } \alpha \in \operatorname{Cn}\left(B^{\prime} \cup\{\beta\}\right) \text { for some } B^{\prime} \subseteq B &
\end{array}
$$

Here, we briefly describe some of these postulates, and for further details see (Hansson, 1999). The relative closure axiom means that the contraction function should not remove a sentence from the original belief base that is (still) implied by the contracted set. The uniformity axiom claims that if two sentences are implied by precisely the same subsets of $B$, then contracting by one of the sentences should be the same as contracting by the other. The relevance axiom is to ensure that if an item is removed from $B$, then there is some reason or rationale for doing so. Both relevance and core-retainment require that $\beta$ contributes to $B$ implying $\alpha$. They do so by identifying $B^{\prime}$ a subset of $B$ that does not imply $\alpha$, but after adding $\beta$ to it, $B^{\prime} \cup\{\beta\}, \alpha$ is implied. The only difference between relevance and core-retainment is the claim that $B \div \alpha$ is a subset of $B^{\prime}$, which is required in relevance and is absent in core-retainment. So, we are "weakening" the requirement that is being defined in the two postulates when we delete it to form core-retainment.

As we will see next, different combinations of the contraction axioms specify different contraction functions. A contraction operation needs to at least satisfy success and inclusion, Hansson explains (1999). That is, the result of a contraction operation should be a subset of the original belief base that does not imply the sentence to be contracted if it is not a tautology.

Definition 2 (Hansson, 1999). An operator $\div$ for a set $B$ is an operator of contraction if and only if it satisfies success and inclusion.

\subsubsection{Belief CONTRACTION CONSTRUCTIONS}

Belief contraction postulates, such as those in the previous section, provide the formal conditions that a belief change operator must satisfy. On the other hand, there may be different ways to construct belief change functions that satisfy different sets of these postulates. Here we consider some important constructions of contraction functions that are relevant to our work.

Partial Meet Contraction For constructing contraction functions it is useful to determine maximal subsets of a belief base $B$ that do not entail a given sentence $\alpha$. Such a maximal non-implying subset of $B$ is called a remainder. Typically, for a given $B$ and $\alpha$, there is more than one remainder, and the collection of all such remainders is called a remainder set. It should be easy to see that any remainder can be accepted as the result of a contraction operation, which, by definition, does not imply $\alpha$. Indeed, since no remainder in a remainder set implies $\alpha$, the intersection of any number 
of them (i.e., any subset of the remainder set) also does not imply $\alpha$, and can be considered to be a possible result of contracting $B$ by $\alpha$. A contraction function $\div$ built this way is called a partial meet contraction. The results of such a construction method clearly will not be unique in the general case, and so the notion of a selection function is introduced to choose the "best" remainders. Partial meet contraction functions have been shown to be axiomatically characterized by $(\div 1)-(\div 6)$ for belief sets (Alchourrón, Gärdenfors \& Makinson, 1985). They are also characterized by success, inclusion, relevance and uniformity for belief bases (Hansson, 2003).

Theorem 3 (Alchourrón, Gärdenfors \& Makinson, 1985). An operator $\div$ for a set $B$ is a partial meet contraction function if and only if $\div$ satisfies the basic postulates for contraction $(\div 1)-(\div 6)$.

Kernel Contraction Kernels are also useful tools in constructing belief change operators for belief bases. While a remainder is a maximal subset of a set of beliefs that do not entail a given formula $\alpha$, a kernel is a minimal subset of a belief base that entails a given formula $\alpha$, which is called an $\alpha$-kernel. A kernel set is the set of all possible $\alpha$-kernels for a belief base $B$. A kernel contraction function removes from $\mathrm{B}$ at least one formula from each of the $\alpha$-kernels. The resulting remaining set will not entail $\alpha$ because each of the $\alpha$-kernels was a minimal set. The postulates success, inclusion, core-retainment and uniformity characterize kernel contraction (Hansson, 1994). While partial meet contraction satisfies relevance, kernel contraction satisfies core-retainment which is a looser constraint. This makes kernel contraction more general than partial meet contraction (Hansson, 1999).

Theorem 4 (Hansson, 1994). The operator $\div$ for $B$ is a kernel contraction if and only if it satisfies success, inclusion, core-retainment and uniformity.

Saturated Kernel Contraction Indeed, kernel contraction constitutes a very general class of belief contraction functions (Hansson, 1999). Saturated kernel contraction is a subset of this class, which also satisfies relative closure.

Theorem 5 (Hansson, 1994). The operator $\div$ for $B$ is a saturated kernel contraction if and only if it satisfies success, inclusion, core-retainment, uniformity and relative closure.

Saturated kernel contraction exhibits a rather interesting property: it is a reversible generalization of partial meet contraction for belief bases, meaning that in the special case where a belief base $B$ is closed, $B=\mathrm{Cn}(B)$, saturated kernel contraction is equivalent to partial meet contraction (Hansson, 1994).

Theorem 6 (Hansson, 1994). Let B be a belief set. Then an operation is a saturated kernel contraction for $B$ if and only if it is a partial meet contraction for $B$.

\subsection{Epistemic Entrenchment}

Regarding construction of contraction operators, a fundamental intuition is that some of our beliefs are more epistemically entrenched than others, making them harder to give up in a contraction or revision. Based on this intuition, Gärdenfors introduced a notion of epistemic entrenchment, and defined the following properties of an order relation, $\leq$, between formulas using five axioms, where 
$\alpha \leq \beta$ is read as " $\alpha$ is at least as epistemically entrenched as $\beta$ " (Gärdenfors, 1988):

(EE1) If $\alpha \leq \beta$ and $\beta \leq \delta$ then $\alpha \leq \delta$

(transitivity)

(EE2) If $\alpha \vdash \beta$ then $\alpha \leq \beta$

(dominance)

(EE3) $\alpha \leq(\alpha \wedge \beta)$ or $\beta \leq(\alpha \wedge \beta)$

(conjunctiveness)

(EE4) If $K \nvdash \perp$ then $\alpha \notin K$ iff $\alpha \leq \beta$ for all $\beta \in \mathcal{L}$

(minimality)

(EE5) If $\beta \leq \alpha$ for all $\beta$ then $\vdash \alpha$

(maximality)

Using these principles of entrenchment, Gärdenfors and Makinson (1988) studied the relation between epistemic entrenchment ordering and belief contraction, and showed that the two are connected by the following conditions:

$\alpha \leq \beta$ iff $\alpha \notin K \div(\alpha \wedge \beta)$ or $\vdash(\alpha \wedge \beta)$

$\beta \in K \div \alpha$ iff $\beta \in K$ and either $\vdash \alpha$ or $\alpha<(\alpha \vee \beta)$

Based on their results, given an epistemic entrenchment relation $\leq$ that satisfies postulates (EE1)-(EE5), a contraction operator $\div$ can be constructed, via $C \div{ }_{G}$, that satisfies $(\div 1)-(\div 8)$. Conversely, given a contraction operator $\div$ satisfying $(\div 1)-(\div 8)$, an epistemic entrenchment relation $\leq$ can be constructed, via $\mathrm{C} \leq$, that satisfies $(\mathrm{EE} 1)-(\mathrm{EE} 5)$.

\subsection{Belief Change and Dependence}

Fariñas del Cerro and Herzig (1996) formalized the notion of a dependence relation and its connection with belief change using an approach similar to that of Gärdenfors and Makinson (1988) for epistemic entrenchment. To formalize dependence relations, they investigate a binary relation $\leadsto$ on formulas. $\alpha \leadsto \beta$ reads as " $\beta$ depends on $\alpha$ " (or equivalently " $\alpha$ is relevant to $\beta$ "). An independence relation, then, is denoted by $\not$, which is the complement of $\leadsto$, so $\alpha \not \rightarrow \beta$ reads as " $\beta$ is independent of $\alpha$ " (or " $\alpha$ is irrelevant to $\beta$ "). For sake of brevity, we sometimes refer to "dependence relation" just as "dependence" throughout this paper. FH provide the following axiomatization of dependence. $^{3}$

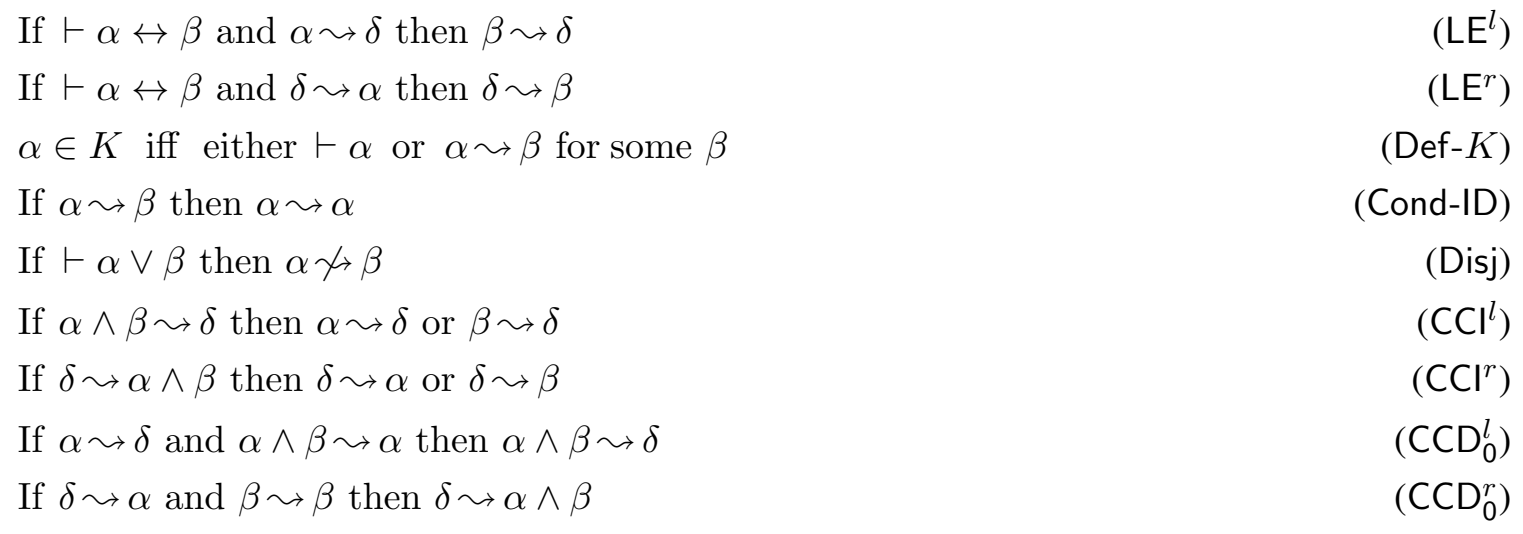

3. FH use " $\alpha \leftrightarrow \beta$ " for $\mathrm{LE}^{l}$ and $\mathrm{LE}^{r}$, which we have changed to " $\vdash \alpha \leftrightarrow \beta$ " here. That is, for these axioms, $\alpha$ and $\beta$ have to be logically equivalent, and not just accidentally have the same truth value. 
The following are two derivable principles:

If $\alpha \leadsto \delta$ and $\beta \leadsto \delta$ then $\alpha \wedge \beta \leadsto \delta$.

The postulates $\mathrm{LE}^{l}$ and $\mathrm{LE}^{r}$, which are symmetric counterparts, are standard postulates for syntax independence. ${ }^{4}$ A more intuitive equivalent form of $\mathrm{LE}^{l}$ is "if $\alpha \leftrightarrow \beta$ then $\alpha \leadsto \delta$ iff $\beta \leadsto \delta$," and similarly for $\mathrm{LE}^{r}$, "if $\alpha \leftrightarrow \beta$ then $\delta \sim \alpha$ iff $\delta \sim \beta$." The next postulate Def- $K$ is the only one that involves $K$. This is important for providing the link between dependence and belief change. Just as an AGM belief contraction operator $\div$ is with respect to some belief set $K$, FH's dependence relation $\leadsto$ is also with respect to some $K$. Assume $\alpha \in K$. In one case, $\alpha$ may be tautological $\vdash \alpha$ because $K$ is deductively closed. In the other case, $\alpha$ is a contingent truth in $K, \nvdash \alpha$, as such its truth has to be inferentially relevant to some (contingent) formula $\beta$ (where $\beta$ could be $\alpha$ ), so $\alpha \leadsto \beta .^{5}$ Def- $K$ makes the correspondence between a dependence relation $\leadsto$ and a belief set $K$ explicit. Cond-ID states that if the truth value of $\alpha$ is inferentially relevant to any formula $\beta, \alpha \sim \beta$, then neither $\alpha$ nor $\beta$ is tautological and as such their truth values are each trivially inferentially relevant to itself, $\alpha \leadsto \alpha$ (and $\beta \leadsto \beta$ ). On the flip side, the truth value of a tautological formula is not inferentially relevant to itself, $\alpha \not \rightarrow \alpha$, nor is it relevant to any other formula $\beta, \alpha \not \rightarrow \beta$. The postulate Disj ensures that a formula and its negation are always independent, $\alpha \not \neg \neg \alpha$. Also, for $\alpha \leadsto \beta$, Disj does not allow $\alpha$ or $\beta$ to be a tautology. FH represent Keynes' CCD and Gärdenfors's $\mathrm{CCl}$ by $\mathrm{CCD}^{l}$ and $\mathrm{CCl}^{l}$, respectively. The symmetric counterparts, $\mathrm{CCD}^{r}$ and $\mathrm{CCl}^{r}$, are also needed because $\leadsto$ is not necessarily symmetric. Instead of directly using $\mathrm{CCD}^{l}$ and $\mathrm{CCD}^{r}$ as postulates, although they remain derivable, FH respectively use the stronger $\mathrm{CCD}_{0}^{l}$ and $\mathrm{CCD}_{0}^{r}$ postulates.

The most constrained form of FH's dependence relation, which uses all the postulates that they introduce, is defined as follows:

Definition 7 (FH). A relation $\leadsto$ is a dependence relation if and only if it satisfies the axioms $\mathrm{LE}^{l}$, $\mathrm{LE}^{r}, \mathrm{CCl}^{l}, \mathrm{CCl}^{r}$, Def- $K$, Cond-ID, Disj, $\mathrm{CCD}_{0}^{l}$ and $\mathrm{CCD}_{0}^{r}$.

As their guiding principle for studying the relationship between dependence and belief change, FH use Gärdenfors's preservation criterion (GPC), which requires that beliefs independent of the changed belief should remain intact in the revised state of belief. For example, if $\beta \in K$ to begin with, but $\beta \notin K \div \alpha$, then we can say that $\beta$ depends on $\alpha$, or $\alpha \leadsto \beta$. $^{6}$

As Gärdenfors and Makinson (1988) did for the case of epistemic entrenchment, FH introduce two conditions to provide the connection between dependence and contraction, namely, Cond $\sim$ and Cond $\div$.

$\alpha \leadsto \beta$ iff $\beta \in K$ and $\beta \notin K \div \alpha$.

(Cond $\leadsto)$

This equivalence condition allows one to define $\leadsto$ based on a given AGM contraction function $\div$ for belief set $K$.

4. Note that $\leadsto$ is a top-level connective. Thus, for example, we never write $(\alpha \leadsto \beta) \wedge(\delta \sim \theta)$; instead we say $(\alpha \leadsto \beta)$ and $(\delta \sim \theta)$. Therefore, $\alpha \wedge \beta \sim \delta$ is interpreted as $(\alpha \wedge \beta) \sim \delta$ and not as $\alpha \wedge(\beta \sim \delta)$.

5. It may also help to look at Def- $K$ in light of GPC. As FH put it, if $\alpha$ is a contingent truth in $K, K$ is contractable by $\alpha$; i.e., $K \div \alpha$ is smaller than $K$. That is, there is some $\beta$ such that $\beta \in K$ but $\beta \notin K \div \alpha$.

6. Technically the $\leadsto$ symbol should be subscripted as $\sim K$, but because the dependence is clear and to avoid clutter, the subscript is dropped. Of course, one could say exactly the same thing about the $\leq$ symbol of epistemic entrenchment. 
Theorem $8(\mathrm{FH})$. Given two relations $\sim$ and $\div$ such that Cond $\sim$ holds, if $\div$ is an AGM contraction function, then $\leadsto$ is a dependence relation.

The other of FH's conditions allows one to define an AGM contraction function $\div$, given a dependence relation $\leadsto$.

$\beta \in K \div \alpha$ iff either $\vdash \beta$ or $(\beta \leadsto \beta$ and $\alpha \not \nrightarrow \beta)$.

(Cond $\div$ )

An AGM contraction function $\div$ is defined with respect to a belief set $K$. Thus, any $\div$ obtained via Cond $\div$ also requires an associated $K$ to be specified. For this purpose, FH provide the following definition for the belief set $K_{\sim}$ :

$K \leadsto=\{\alpha \mid \vdash \alpha$ or $\alpha \leadsto \beta$ for some $\beta\}$.

To avoid clutter, FH simply use $K$ to refer to $K \leadsto$ afterwards. The next theorem defines a contraction function, given a dependence relation using Cond $\div$.

Theorem 9 (FH). Given two relations $\leadsto$ and $\div$ such that Cond $\div$ holds, if $\leadsto$ is a dependence relation then $\div$ is an AGM contraction function.

As the last step, FH need to complete the link between AGM contraction and dependence using a characterization theorem to state that for any two arbitrary relations $\sim$ and $\div$ that satisfy Cond $\leadsto$, $\div$ is an AGM contraction function iff $\sim$ is a dependence relation. To achieve this, however, it turns out that they first have to make the following assumption:

Remark 10. In order to establish an axiomatic characterization based on Cond $\leadsto$, it is assumed that the relation $\div$ satisfies inclusion, $K \div \alpha \subseteq K$.

Theorem $11(\mathrm{FH})$. Let two relations $\sim$ and $\div$ be such that $\div$ satisfies inclusion and that Cond $\leadsto$ holds. Then $\div$ is an AGM contraction function if and only if $\leadsto$ is a dependence relation.

FH also provide a characterization theorem for a weaker dependence relation than the one we cited in Definition 7.

Theorem $12(\mathrm{FH})$. Let two relations $\leadsto$ and $\div$ be such that $\div$ satisfies inclusion and that Cond $\leadsto$ holds. Then $\div$ is a basic AGM contraction function satisfying $(\div 1)-(\div 6)$ if and only if $\leadsto$ is a dependence relation satisfying $\mathrm{LE}^{l}, \mathrm{LE}^{r}, \mathrm{CCI}^{r}$, Def- $K$, Cond-ID, Disj and $\mathrm{CCD}_{0}^{r}$.

This completes our discussion of the foundations of FH's work.

\section{Base Dependence}

Gärdenfors' preservation criterion lays the foundation of the present work, as it did with FH. Our work is a further attempt to connect notions of dependence and belief change, but using belief bases instead of belief sets. 


\subsection{Overview and Problem Definition}

As discussed above, belief bases are a generalization of belief sets. Hence, it seems natural to anticipate that base dependence also be a generalization of FH's dependence relation. Furthermore, ideally we would like to identify the conditions under which base dependence will be a reversible generalization of dependence. That is, if a base dependence relation is with respect to a a belief base that is a belief set, then the base dependence relation reduces to FH's dependence.

Finally, one significant feature of FH's formalism is that it adheres to Keynes's CCD and Gärdenfors' CCI. This is a characteristic of FH's dependence that we would like to preserve, while we generalize it to base dependence. It will turn out that our base dependence formalism meets a third principle, CCDF, that implies both CCD and CCI.

\subsubsection{Characteristics of an ANTICIPATEd Solution}

We suggest that a formalization of Gärdenfors' preservation criterion that involves belief bases instead of belief sets should still retain the general scheme of FH's work. More specifically, we will need an axiomatization of base dependence, as well as a suitable corresponding belief base contraction. We will also need a condition, similar to FH's Cond $\leadsto$, that allows construction of a base dependence given a base contraction, and another condition, similar to FH's Cond $\div$, that allows construction of a base contraction using a base dependence.

These characteristics are illustrated in Figure 3 in $\S 5$. The remainder of this paper is to establish the background concepts and theorems necessary to justify the diagram in Figure 3.

\subsection{Base Dependence Relation}

The meaning of "dependence" in base dependence relation is the same as what Fariñas and Herzig (and Gärdenfors) studied, and refers to the dependence or relevance of logical statements towards one another. Using their notation, we read $\alpha \leadsto \beta$ as " $\beta$ depends on $\alpha$ " or "doubting in $\alpha$ leads to doubting in $\beta$." We sometimes refer to "base dependence relation" just as "base dependence" for the sake of brevity.

In FH's study, dependence may only exist between (contingent) sentences from $K$ :

If $\alpha \leadsto \beta$ then $\alpha \in K$ and $\beta \in K$.

If $B$ is a belief base for $K$, i.e. $K=\operatorname{Cn}(B)$, then we have:

If $\alpha \leadsto \beta$ then $\alpha \in \operatorname{Cn}(B)$ and $\beta \in \operatorname{Cn}(B)$.

One way to generalize the dependence relation $\leadsto$ is to make either $\alpha$ or $\beta$ be from $B$ instead of $\operatorname{Cn}(B)$. Therefore, our sought-for base dependence should somehow involve formulas explicitly mentioned in a belief base, thus the name.

Using $\leadsto$ to denote base dependence, we read $\alpha \leadsto \beta$ as " $\beta$ base-depends on $\alpha$. " In a more cognitively-oriented way of putting it, $\alpha \leadsto \beta$ means that "doubting $\alpha$ would lead to doubting $\beta$," which is the same as $\alpha \leadsto \beta$, but $\alpha \leadsto \beta$ also implies that $\alpha$ or $\beta$ or both are formulas in the belief base. Now, we need to decide which one of these three alternatives should be the case.

If $\alpha \leadsto \beta$ then $\alpha \in B$ and $\beta \in \operatorname{Cn}(B)$

If $\alpha \leadsto \beta$ then $\alpha \in \operatorname{Cn}(B)$ and $\beta \in B$

If $\alpha \leadsto \beta$ then $\alpha \in B$ and $\beta \in B$. 
We will show in $\S 3.6$ that indeed (3b) is the most general alternative. It means "doubting in $\alpha$ leads to doubting in $\beta$ from the belief base." It allows us to study how the statements in the belief base, $\beta \in B$, depend on or are susceptible to changing our beliefs about other statements $\alpha \in \mathcal{L}$.

Therefore, although the alternatives ( $3 a$ ) and (3c) remain open and may be found useful in other studies, we proceed with the most general alternative (3b), and from now on we assume $\alpha \leftrightharpoons \beta$ requires that $\beta \in B$ :

If $\alpha \leadsto \beta$ then $\beta \in B$.

It turns out that (4) does not need to be explicitly specified as an independent axiom. Rather, it will be implied by other axioms and conditions for base dependence which will be put forward in the upcoming sections (e.g., Def- $B$ and Cond $\leadsto$ ). The following provides an example base dependence relation:

Example 13. Assume Mary believes that $p, q$ and $q \rightarrow r$; i.e., $B=\{p, q, q \rightarrow r\}$. She draws the implication that $\mathrm{r}$ holds, as it is entailed by $q$ and $q \rightarrow r$. Now, say, for some reason, she starts to doubt that $r$ is true. Consequently, this leads her to also doubt either $q$, or $q \rightarrow r$, or even both. Thus

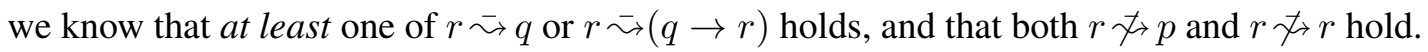

In the above example, although we know that at least one of $r \leadsto q$ or $r \leftrightharpoons(q \rightarrow r)$ holds, we do not know which one(s). We need more information to be able to determine that. Such extra-logical information is provided in the following example.

Example 14. Assume as in Example 13, Mary believes the same $B=\{p, q, q \rightarrow r\}$, but this time she also knows that if she ever doubts $r$, then she would prefer doubting $q$, but not $q \rightarrow r$. Thus, for her, believing $q$ depends on $r, r \leadsto q$, but believing $q \rightarrow r$ does not, $r \not \rightarrow(q \rightarrow r)$. That is, in the event that Mary stops believing $r$, she would also stop believing $q$, but then according to Gärdenfors' preservation criterion, she would retain the rest of her beliefs: $B^{\prime}=\{p, q \rightarrow r\}$. In other words, we have $B^{\prime}=B-r$. The dependencies between beliefs can determine how they are revised (and vice versa).

\subsection{Basic Postulates of Base Dependence}

A goal of this work is to provide an axiomatization of base dependence as a generalization of FH's dependence. It turns out that some of the base dependence axioms closely resemble their dependence axioms (e.g. Cond-ID ${ }^{B}$ ), and some remain valid and derivable but are no longer needed as axioms (e.g. $\left.\operatorname{Disj}^{B}\right)$. However, there are also some other axioms that have been offered for base dependence (e.g. redundancy and modularity) which are not similar to any of the dependence axioms.

Let us start by providing a simplifying notation $\operatorname{Taut}(B)$ to represent tautologies present in a belief base $B$.

Definition 15. Given a belief base $B$, Taut $(B)$ is the set of all tautologies in $B$ : $\operatorname{Taut}(B)=\{\beta \mid$ $\beta \in B$ and $\vdash \beta\}$.

Clearly it holds that $\operatorname{Taut}(B) \subseteq B$. One important usage of this definition is to help handling tautologies in base dependence axioms. Such axioms are primarily concerned with contingencies, but they have to also deal with tautologies in exceptional cases. 
We now offer the following basic postulates for base dependence.

$\beta \in B$ iff either $\beta \in \operatorname{Taut}(B)$ or $\alpha \leadsto \beta$ for some $\alpha$.

(Def-B)

If $\alpha \leadsto \beta$ then $\beta \approx \beta$.

(Cond-ID ${ }^{B}$ )

If $\alpha \in \operatorname{Cn}\left(B^{\prime}\right)$ iff $\beta \in \operatorname{Cn}\left(B^{\prime}\right)$ for all $B^{\prime} \subseteq B$

(conjugation)

then $\alpha \leadsto \delta$ iff $\beta \approx \delta$.

If $\alpha \leadsto \beta$

(contribution)

then $\alpha \notin \operatorname{Cn}\left(B^{\prime}\right)$ and $\alpha \in \operatorname{Cn}\left(B^{\prime} \cup\{\beta\}\right)$ for some $B^{\prime} \subseteq B$.

If $\alpha \in \operatorname{Cn}\left(B^{\prime}\right)$ and $B^{\prime} \subseteq B$

(modularity)

then either $\vdash \alpha$ or $\alpha \leadsto \beta$ for some $\beta \in B^{\prime}$.

If $\beta \in \operatorname{Cn}\left(B^{\prime}\right)$ and $B^{\prime} \subseteq B$

(redundancy)

then either $\alpha \not \Varangle \beta$ or $\alpha \leadsto \delta$ for some $\delta \in B^{\prime}$.

A brief description of these axioms is as follows:

(Def- $B$ ) To say that $\beta$ is in the belief base, $\beta \in B$, is equivalent to saying either that $\beta$ is a tautology in the belief base, $\beta \in \operatorname{Taut}(B)$, or that it is a contingency in the belief base. The contingent truth of $\beta$ then has to be inferentially relevant to some (contingent) formula $\alpha$ (where $\alpha$ could be $\beta$ ), so $\alpha \leadsto \beta$.

(Cond-ID ${ }^{B}$ ) The inferential relevance between $\alpha$ and $\beta$ means that neither is a tautology. Also, $\beta$ is in the belief base because $\alpha \leadsto \beta$. Thus, $\beta$ is a contingency in the belief base, or $\beta \leadsto \beta$.

(conjugation) When $\alpha$ and $\beta$ are logically equivalent and $\delta \in B$, there is inferential relevance between $\alpha$ and $\delta$ if and only if there is inferential relevance between $\beta$ and $\delta$ : If $\vdash \alpha \leftrightarrow$ $\beta$ then $\alpha \doteqdot \delta$ iff $\beta \leftrightharpoons \delta$. This makes sense not only for $\alpha$ and $\beta$, but also for the formulas that are true just because $\alpha$ or $\beta$ are true. For example, assume that $\alpha \vee \theta$ holds just because $\alpha$ holds. Then we can say: If $\vdash \alpha \leftrightarrow \beta$ then $(\alpha \vee \theta) \leadsto \delta$ iff $\beta \leadsto \delta$. All these cases can be captured by the axiom's more general antecedent: $\alpha \in \operatorname{Cn}\left(B^{\prime}\right)$ iff $\beta \in \operatorname{Cn}\left(B^{\prime}\right)$ for all $B^{\prime} \subseteq$ $B$.

(contribution) This axiom says that if $\beta \in B$ is inferentially relevant to $\alpha$ then $\beta$ must contribute to the justification of $\alpha$, or, in other words, contracting $B$ by $\alpha$ requires retracting $\beta$ from the belief base.

(modularity) Consider a subset $B^{\prime} \subseteq B$ that implies $\alpha$, so that $\alpha \in \operatorname{Cn}\left(B^{\prime}\right)$. This could be because $\alpha$ is a tautology. But when $\forall \alpha$, there is some $\beta$ from the same subset $B^{\prime}$ that base-depends on $\alpha$.

(redundancy) To consider the principal case of redundancy, assume that: $\beta \in \operatorname{Cn}\left(B^{\prime}\right)$ and $B^{\prime} \subseteq B$ and $\alpha \leadsto \beta$. In the case where $\beta \in B^{\prime}$, it trivially follows from the fact that $\alpha \leftrightharpoons \beta$ that there is some $\delta \in B^{\prime}$ such that $\alpha \approx \delta$. In the other case where $\beta \notin B^{\prime}$, there is some redundancy in the belief base $B$ because on the one hand $\beta \in B$ (as $\alpha \leadsto \beta$ ), and on the other hand there is $B^{\prime} \subseteq B$ such that $\beta \notin B^{\prime}$ but $\beta \in \operatorname{Cn}\left(B^{\prime}\right)$. Thus, in order for $\alpha \leadsto \beta$ to hold, $\alpha \leftrightharpoons \delta$ must also hold for at least one formula $\delta \in B^{\prime}$. Thus, the redundancy postulate captures both possible cases. 
Example 16. Assume that $B=\{p \leftrightarrow q, p \vee q, p\}$, and that $\doteqdot$ is a relation such that $(p \wedge q) \doteqdot(p \vee q)$ and, for reductio, that $(p \wedge q) \not \rightarrow p$ holds. We show that $\leadsto$ violates redundancy. Let $B^{\prime}=\{p\}$. Clearly $B^{\prime} \subseteq B$ and $p \vee q \in \mathrm{Cn}\left(B^{\prime}\right)$, and, by assumption, $(p \wedge q) \leadsto(p \vee q)$. Thus, by redundancy, $(p \wedge q) \leadsto \delta$ for some $\delta \in B^{\prime}$. Since $B^{\prime}=\{p\}, \delta$ has to be $p$, so $(p \wedge q) \leadsto p$. This contradicts the initial assumption that $(p \wedge q) \not>p$.

Furthermore, the following principles are counterparts to FH's axioms Disj and $\mathrm{LE}^{l}$, respectively. In our study, $\operatorname{Disj}^{B}$ and $\mathrm{LE}^{B}$ turn out to be derivable from the above-mentioned axioms.

If $\vdash \alpha \vee \beta$ then $\alpha \not \rightarrow \beta$.

If $\vdash \alpha \leftrightarrow \beta$ and $\alpha \leadsto \delta$ then $\beta \leadsto \delta$.

Theorem 17. If a relation $\leadsto$ satisfies conjugation, then it also satisfies $\mathrm{LE}^{B}$.

Theorem 18. If a relation $\leadsto$ satisfies contribution, then it also satisfies $\operatorname{Disj}^{B}$.

Thus, conjugation implies $\mathrm{LE}^{B}$, and contribution implies $\mathrm{Disj}^{B}$, by Theorems 17 and 18 , respectively.

Definition 19. A relation $\leadsto$ is a base dependence if and only if it satisfies the axioms Def- $B$, Cond-ID ${ }^{B}$, conjugation, contribution and modularity.

It turns out that there is an important sub-class of $\leadsto$ relations defined above that also satisfy redundancy. For reasons that will become clear in the upcoming sections, we refer to this subclass as strong base dependence:

Definition 20. A relation $\leadsto$ is a strong base dependence if and only if it is a base dependence that satisfies redundancy.

Notice that so far we have not specified any criteria on how to handle conjunctions, which we will do in $\S 4.3$.

\subsection{Mutual Construction of Base Contraction and Base Dependence}

In this subsection, we aim to mutually connect the notions of base dependence and base contraction using Gärdenfors' preservation criterion as a guideline.

\subsubsection{From Base Contraction to Base Dependence}

As discussed earlier, FH use Cond $\leadsto$ to construct a dependence relation via a given AGM contraction function. Cond $\leadsto$ can be straightforwardly transformed to an equivalent base-generated representation. That is, if $K=\operatorname{Cn}(B)$ and $\div$ is a base-generated contraction function (which exists for any given AGM contraction as Hansson, 1993, shows), then Cond $\sim$ can be stated as follows:

$\alpha \sim \beta$ iff $\beta \in \operatorname{Cn}(B)$ and $\beta \notin \operatorname{Cn}(B \div \alpha)$.

(Cond $\leadsto$ )

Note that we have reused the same name "Cond $\sim$ " above since this is only an alternative representation. This new, base-generated representation makes it easier to compare and contrast FH's Cond $\leadsto$ with the corresponding conditions for belief bases that will be introduced in this section and in $\S 3.5$. 
If $\alpha \leadsto \beta$ holds then $\beta$ is retracted as a result of contracting by $\alpha$, so $\beta \in[\operatorname{Cn}(B) \backslash \operatorname{Cn}(B \div \alpha)]$. For base dependence, we assume that (4) holds: if $\alpha \approx \beta$ then $\beta \in B$. Thus, it is intuitively appealing to say that if $\alpha \leadsto \beta$ holds, then $\beta$ will be retracted from $B$ as a result of contracting by $\alpha$, and so $\beta \in[B \backslash B \div \alpha]$. With this intuition in mind, we propose the following to correspond to FH's Cond $\leadsto$ :

$\alpha \leadsto \beta$ iff $\beta \in B$ and $\beta \notin B \div \alpha$.

(Cond $\leadsto)$

Notice that in common with FH, if $\alpha$ is a tautology, no formula contributes to its truth, and $\alpha \leadsto \beta$ cannot hold for any $\beta$. Also, if $\beta \notin B$, then $\alpha \leadsto \beta$ cannot hold for any $\alpha$ by definition. Therefore, both $\beta \leadsto \beta$ and $\beta \leadsto \beta$ imply that $\beta$ is contingent, and the latter additionally implies that $\beta \in B$. This will prove useful in the next section when constructing contraction functions using (base) dependence, via Cond $\div$ and Cond $\overline{\div}$.

Also note that we do not presume that Cond $\leadsto$ is the only way to generalize FH's Cond $\leadsto$ for belief bases. Indeed, we will discuss other ways of doing so in $\S 3.5$.

\subsubsection{From Base Dependence to Base Contraction}

We now reconstruct belief bases, given base dependence relations. We saw in $\S 2.5$ that FH provide (1):

$K_{\sim}=\{\alpha \mid \vdash \alpha$ or $\alpha \leadsto \beta$ for some $\beta\}$.

Note that we could swap the role of $\alpha$ and $\beta$ above and obtain the same results:

$K_{\sim}=\{\beta \mid \vdash \beta$ or $\alpha \leadsto \beta$ for some $\alpha\}$.

Similarly, a base dependence relation $\precsim$ is associated with a belief base $B$. Thus, it should be possible to recreate the associated belief base $B$ via a given $\leadsto$ relation:

$B_{\approx}=\{\beta \mid \alpha \leadsto \beta$ for some $\alpha\}$.

Note, however, that one caveat here is that $B_{\approx}$ will not contain any tautologies, not even any of the tautologies that may be in $B$. Still, $B$ and $B \lesssim$ are equivalent for most practical purposes. Also, their closure is obviously equivalent: $\operatorname{Cn}\left(B_{\leadsto}\right)=\operatorname{Cn}(B)$.

If in addition to the base dependence relation $\leadsto$, we assume that we are also given the tautologies in the belief base, Taut $(B)$, then we can have the following, which guarantees that $B_{\varlimsup}=B$ :

$B_{\bar{\varpi}}=\{\beta \mid \beta \in \operatorname{Taut}(B)$ or $\alpha \leadsto \beta$ for some $\alpha\}$.

In the rest of this paper, we assume that $B_{\hookrightarrow}=B$. In the extreme case, i.e., if $\operatorname{Taut}(B)$ is not given, there could be tautologies in $B$ but only $\leadsto$ is given, so the tautologies in $B$ are not present in $B=$.

For Cond $\overline{\div}$, we again start with FH's Cond $\div$. As in the case of Cond $\leadsto$, we present a straightforward transformation to the equivalent base-generated operation. Again, we reuse the equation's name, "Cond $\div$ ":

$\beta \in \operatorname{Cn}(B \div \alpha)$ iff either $\vdash \beta$ or $(\beta \sim \beta$ and $\alpha \not \neg \beta)$.

(Cond $\div$ ) 
Cond $\div$ says $\beta \in \operatorname{Cn}(B \div \alpha)$ means either that $\beta$ is a tautology, or that $\beta$ is a contingent truth, $\beta \sim \beta$, but that contraction by $\alpha$ does not lead to retraction of $\beta$, thus implying that $\alpha \not \neg \beta$.

To adapt this for belief bases, we need the following: $\beta \in B \div \alpha$ means either that $\beta$ is a tautology in $B, \beta \in \operatorname{Taut}(B)$, or that $\beta$ is a contingent truth in $B, \beta \leadsto \beta$, but that contraction by $\alpha$ does not lead to retraction of $\beta$ from $B, \alpha \not \star \beta$ :

$\beta \in B \div \alpha$ iff either $\beta \in \operatorname{Taut}(B)$ or $(\beta \leftrightharpoons \beta$ and $\alpha \not \nrightarrow \beta)$.

(Cond $\overline{\div})$

\subsection{Different Types of Base Dependence Construction}

In contrast with FH's dependence relations for belief sets, there is more than one way to construct base dependence relations. We have already made the decision to define base dependence relations such that $\alpha \leadsto \beta$ implies $\beta \in B$ (as opposed to alternative formalisms of base dependence relations such that they imply $\alpha \in B$ or both $\alpha, \beta \in B$ ). However, even after specifying that "if $\alpha \leadsto \beta$ then $\beta \in B$," there still remain different "kinds" of base dependence to consider.

Throughout this section, we use Figure 1 to provide a running example to help illustrate different existing or new concepts. To start with the simpler case, consider FH's use of Cond $\leadsto$ to construct a dependence relation using a given AGM contraction function. An example application of Cond $\leadsto$ is depicted in Figure 1a. It shows a belief set $K$ along with some example formulas: $\alpha, \beta, \omega, \delta, \iota$ and $\epsilon$ in $K$. It further shows how contracting $K$ by $\alpha$ also results in retraction of some other formulas: $\beta$ and $\delta$, but not the rest of the formulas. Thus, we conclude by Cond $\sim$ that: $\alpha \leadsto \beta$ and $\alpha \leadsto \delta$, and that $\alpha \not \rightarrow \omega, \alpha \not \triangleleft \iota$ and $\alpha \psi_{\triangleleft} \epsilon$. These results are summarized in the first row of Table 1 .

\subsubsection{Base DePEndence Constructions}

Construction 1: Base Dependence We have already seen the condition Cond $\leadsto$ in $\S 3.4 .1$ :

$\alpha \leadsto \beta$ iff $\beta \in B$ and $\beta \notin B \div \alpha$.

(Cond $\leadsto)$

Figure $1 \mathrm{~b}$ shows a belief base $B$ and its logical closure $\operatorname{Cn}(B)$. Here, some formulas from belief base $B$ have been retracted, namely, $\beta$ and $\omega$, so that $\alpha$ is not implied by the resulting belief base, $\alpha \notin \mathrm{Cn}(B \div \alpha)$. By Cond $\leadsto$, then, we conclude that $\beta$ and $\omega$ base-depend on $\alpha: \alpha \leadsto \beta$ and $\alpha \backsim \omega$. Cond $\leadsto$ maintains its intuitive appeal as a reasonable formalization of GPC. Nevertheless, there remain some subtleties that we will explore next.

Construction 2: Strong Base Dependence Before discussing the next base dependence construct, let us once again consider the base-generated representation of FH's Cond $\leadsto$ construction:

$\alpha \leadsto \beta$ iff $\beta \in \mathrm{Cn}(B)$ and $\beta \notin \operatorname{Cn}(B \div \alpha)$.

(Cond $\leadsto$ )

Now, comparing Cond $\leadsto$ and Cond $\leadsto$ above makes it clear that indeed there is another possible formalization of GPC for belief bases as follows:

$\alpha \hat{\sim} \beta$ iff $\beta \in B$ and $\beta \notin \operatorname{Cn}(B \div \alpha)$.

(Cond $\hat{\sim})$

This provides a stronger condition for base dependence than Cond $\leadsto$ because $B \div \alpha \subseteq \mathrm{Cn}(B \div \alpha)$ by the inclusion property of the Cn operator.

Going back to Figure 1b, we saw that by Cond $\bar{\sim}: \alpha \leadsto \beta$ and $\alpha \leadsto \omega$. However, according to Cond $\hat{\sim}, \beta$ has strong base dependence on $\alpha, \alpha \hat{\sim} \beta$, but $\omega$ does not, $\alpha \hat{\psi} \omega \omega$. When contracting $B$ 
(a)

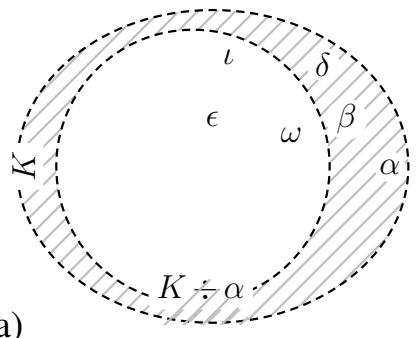

(b)

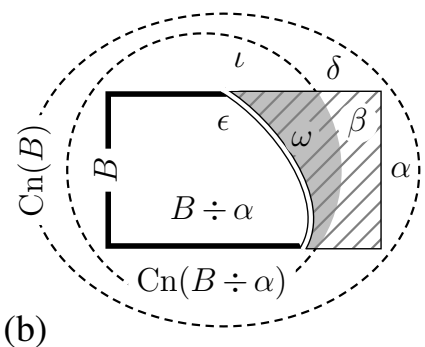

Figure 1: Building (b) base dependence via Cond $\leadsto$ is more complex than (a) dependence via Cond $\rightarrow$. Example formulas $\alpha, \beta, \omega, \delta, \iota, \epsilon$ fall into different subareas. Assume $K=\operatorname{Cn}(B)$.

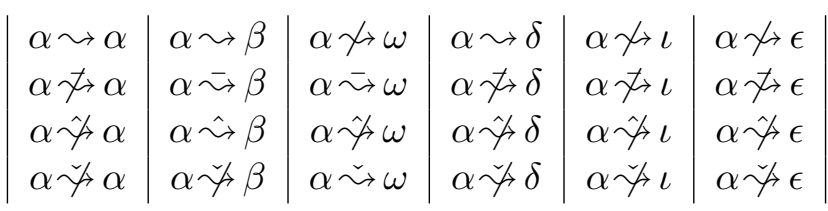

Table 1: Examples of different types of (base) dependence, obtained via Cond $\leadsto$, Cond $\leadsto$, Cond $\hat{\sim}$ and Cond $\sim$, for the illustrated formulas in Figure 1.

by $\alpha, \beta$ is retracted whether we consider the resulting belief base, $B \div \alpha$, or its closure, $\operatorname{Cn}(B \div \alpha)$. This is not the case for $\omega$, which we will study next.

Construction 3: Weak Base Dependence To further investigate the difference between Cond $\leadsto$ and Cond $\hat{\sim}$, observe that $\omega$ is originally in $B$, and it is then retracted as a result of contracting $B$ by $\alpha, \omega \notin B \div \alpha$, but later it is reintroduced as a logical implication of the contracted set, $\omega \in$ $\operatorname{Cn}(B \div \alpha)$. We refer to this non-persistent base dependence of $\omega$ on $\alpha$ as weak base dependence, denoted by $\alpha \leadsto \omega$. On the one hand, $\alpha \approx \omega$ refers to a kind of base dependence in the sense that $\omega$ is removed from the belief base as a result of contracting by $\alpha$. On the other hand, it does not fully capture the concept of dependence because $\omega$ is still implicitly present in the consequences of the contracted set. Thus even though it is a kind of base dependence, it is a weak dependence. Basically a base dependence which is not a strong base dependence is a weak base dependence, which can be specified as follows:

$\alpha \stackrel{\sim}{\beta}$ iff $\beta \in B$ and $\beta \notin B \div \alpha$ and $\beta \in \operatorname{Cn}(B \div \alpha)$.

$($ Cond $\sim$ )

\subsubsection{Connections Among (BAse) Dependence Constructions}

The above-mentioned base dependence constructions as well as FH's dependence construction Cond $\sim$ are all connected. For example, a base dependence between two formulas, $\alpha \leadsto \beta$, is either a strong base dependence, $\alpha \hat{\sim} \beta$, or a weak base dependence, $\alpha \stackrel{\sim}{ } \beta$.

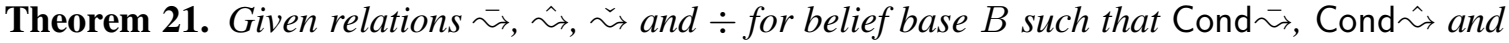
Cond $\sim$ hold respectively, the following also holds: $\quad \alpha \leadsto \beta$ iff $\alpha \dot{\sim} \beta$ or $\alpha \leadsto \beta$ 
The proof is rather straightforward by comparing the right hand sides of the three conditions. Indeed, base dependence and strong base dependence become equivalent when there is no weak base dependence, which is guaranteed when relative closure is satisfied (as also shown by Theorem 29 below).

Theorem 22. Given relations $\vec{\leadsto} \hat{\sim}$ and $\div$ for belief base B such that Cond $\leadsto$ and Cond $\hat{\sim}$ hold respectively and relative closure is satisfied, the base dependence relation $\leadsto$ and the strong base dependence relation $\hat{\sim}$ are equivalent: $\quad \alpha \leadsto \beta$ iff $\alpha \hat{\sim} \beta$.

As a proof sketch, assume $\beta \in B$ and $\beta \in \operatorname{Cn}(B \div \alpha)$. Then, it also holds that $\beta \in B \div \alpha$ by relative closure: $B \cap \operatorname{Cn}(B \div \alpha) \subseteq B \div \alpha$. Thus, by Cond $\sim, \alpha \sim \beta$ does not hold. Then, by Theorem 21, we have $\alpha \stackrel{\sim}{\sim}$ iff $\alpha \stackrel{\sim}{\sim} \beta$.

Finally, we establish the connection between dependence and base dependence. In the presence of relative closure or equivalently in the absence of weak base dependence, base dependence is equivalent to dependence for the formulas in the belief base.

Theorem 23. Given relations $\leadsto, \sim$ and $\div$ for belief base $B$ such that Cond $\leadsto$ and Cond $\leadsto$ hold respectively and relative closure is satisfied, we obtain: $\quad \alpha \leadsto \beta$ iff $\beta \in B$ and $\alpha \leadsto \beta$.

This result paves the way to show next that when $B$ is logically closed, i.e., $B=\mathrm{Cn}(B)$, base dependence and dependence become equivalent. This is depicted in Figure 2.

Theorem 24. Given relations $\leadsto, \leadsto$ and $\div$ for belief base B such that Cond $\leadsto$ and Cond $\leadsto$ hold respectively and closure is satisfied, in the case where $B$ is logically closed, $\leadsto$ reduces to $\leadsto$ : $\alpha \leadsto \beta$ iff $\alpha \leadsto \beta$.

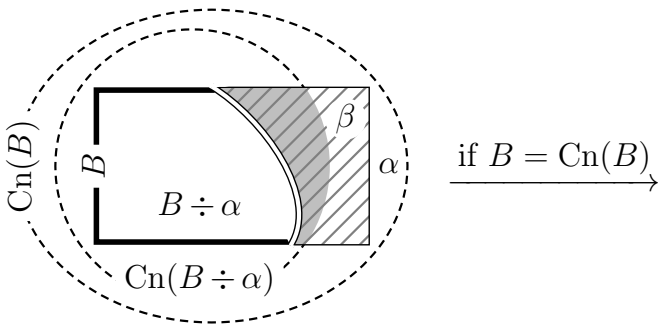

(a) Base dependence for base $B$, which is generally not closed

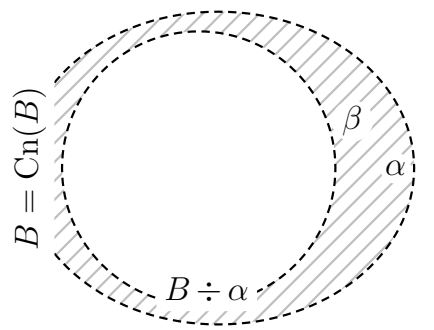

(b) When $B$ is closed though, base dependence reduces to dependence

Figure 2: Dependence is a special case of base dependence.

\subsubsection{Redundancy Resulting in Different Types of Base Dependence}

We are now interested to know the conditions under which there is no weak base dependence because then, for example, we can say when base dependence and strong base dependence are equivalent, based on the previous results in this section. Formally, we define absence of weak base dependence as follows:

Definition 25. Given relations $\leadsto$ and $\div$ for belief base $B$ such that Cond $\check{\sim}$ holds, we say that there is no weak base dependence if and only if $\alpha \ddot{\psi} \beta$ for all formulas $\alpha$ and $\beta$. 
In this section we show that there is a powerful correspondence between two seemingly different concepts: weak base dependence and redundancy of belief bases.

We give the following definition to clarify what redundancy in a belief base means.

Definition 26. $\beta$ is redundant in $B$ with respect to $B^{\prime}$ if and only if $B^{\prime} \subseteq B$ and $\beta \in B$ and $\beta \notin B^{\prime}$ and $\beta \in \operatorname{Cn}\left(B^{\prime}\right)$.

The following theorem shows that weak base dependence exists exactly when some redundant contracted statements are still implied by the remaining statements.

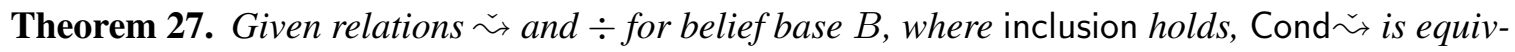
alent to the following: $\quad \alpha \sim \beta$ iff $\beta$ is redundant in $B$ with respect to $B \div \alpha$.

One immediate and interesting implication of this theorem is that weak base dependence cannot occur in a belief base that contains no redundancy.

Corollary 28. Given relations $\leadsto$ and $\div$ for belief base B such that Cond $\sim$ and inclusion hold, the following also holds: $\quad$ if $B$ has no redundancy, then it contains no weak base dependence.

To summarize the results so far, let us consider Definition 25. Note that for any $\beta \notin B$, it trivially holds by Cond $\sim$ that $\alpha \ddot{\psi} \beta$ for all $\alpha$. More interesting instances of absence of weak base dependence can occur when $\beta \in B$. It is a property of the belief base $B$ and/or the contraction operator $\div$ used that determines whether any weak base dependence can exist. By Corollary 28, if belief base $B$ does not contain any redundancy, then there will be no weak base dependence involving any of its formulas. On the other hand, if $B$ contains some redundancy, then, by Theorem 27, existence of weak base dependence will depend on the corresponding contraction function $\div$ (via Cond $\sim$ ), and on how $\div$ handles any redundancy that may exist in the belief base $B$.

The following theorem formally identifies relative closure as the condition under which there does not exist weak base dependence between any given pair of sentences.

Theorem 29. Given relations $\leadsto$ and $\div$ for belief base $B$ such that Cond $\sim$ holds, there is no weak base dependence if and only if relative closure holds for $\div$.

Thus, existence of weak base dependence depends solely on the corresponding contraction operator. A base dependence constructed using a contraction operator that satisfies relative closure cannot be a weak base dependence by Theorem 29, and thus it is a strong base dependence by Theorem 21.

\subsection{Generality of Base Dependence Construction}

In $\S 3$, we considered the following three alternatives for base dependence to generalize FH formalism. We also mentioned that it turns out that the most general alternative is (3b), which we can show now in this section.

If $\alpha \leadsto \beta$ then $\alpha \in B$ and $\beta \in \operatorname{Cn}(B)$

If $\alpha \leadsto \beta$ then $\alpha \in \operatorname{Cn}(B)$ and $\beta \in B$

If $\alpha \leadsto \beta$ then $\alpha \in B$ and $\beta \in B$.

Let us once again consider Gärdenfors's preservation criterion GPC (see $\S 1$ ) as the basis of both FH's and our formalisms. FH's attempt to formalize this intuition is most apparent in Cond $\leadsto$ : 
$\alpha \leadsto \beta$ iff $\beta \in \operatorname{Cn}(B)$ and $\beta \notin \operatorname{Cn}(B \div \alpha)$.

To achieve alternative (3a), FH's Cond $\sim$ can be altered to the following:

$\alpha \leadsto \beta$ iff $\alpha \in B$ and $\beta \in \operatorname{Cn}(B)$ and $\beta \notin \operatorname{Cn}(B \div \alpha)$.

(Cond $\sim_{\alpha}$ )

That is, in contrast with FH's Cond $\sim$, Cond $\sim \alpha$ has to be further constrained by the new conjunct $\alpha \in B$. Whatever the result of this further constraint is, it will produce a subset of FH's formalism and not a generalization of it. Although this subset of FH's work may be interesting for some other study, our goal here has been to generalize their work.

Alternative (3b) can result in either of the following, both of which are studied in our formalism (see $\S 3.5 .1)$ :

$\alpha \leadsto \beta$ iff $\beta \in B$ and $\beta \notin B \div \alpha$.

(Cond $\leadsto)$

$\alpha \stackrel{\sim}{\sim}$ iff $\beta \in B$ and $\beta \notin \operatorname{Cn}(B \div \alpha)$.

(Cond $\hat{\sim})$

For alternative (3c), let us start by considering the above conditions Cond $\leadsto$ or Cond $\hat{\sim}$, which already contain the conjunct $\beta \in B$. They can be further constrained by adding the conjunct $\alpha \in B$. Given that the condition Cond $\leadsto$ is more general than Cond $\hat{\sim}$ (see Theorem 22), it suffices here that we only consider adding the conjunct $\alpha \in B$ to the more encompassing condition Cond $\leadsto$ :

$\alpha \leadsto \beta$ iff $\alpha \in B$ and $\beta \in B$ and $\beta \notin B \div \alpha$.

(Cond $\sim_{\alpha}$ )

A base dependence relation $\leadsto$ produced by Cond $\check{\sim}_{\alpha}$ is a very restricted form of our base dependence. For any non-trivial case where $\alpha$ and $\beta$ are different formulas, Cond $\sim_{\alpha}$ can only be satisfied when there is redundancy in the belief base $B$. That is because Cond $\bar{\sim}_{\alpha}$ requires that retracting $\alpha$ from the belief base $B$ also retracts some other formula $\beta$ from $B$. Given the minimality constraint of rational belief contraction operations, this can happen only if $\beta$ is contributing to implying $\alpha$. Thus, $\alpha$ is not only an explicit member of $B$ but also implied by some other formula(s) in $B$, which means there is some redundancy in $B$. Simply put, Cond $\sim_{\alpha}$ is a more restricted form of Cond $\leadsto$.

In summary, the most general formalism of base dependence may be achieved through the alternative (3b), which will be a superset of the results produced by the other two alternatives (3a) and $(3 \mathrm{c})$.

\section{Belief Change and Base Dependence}

We are now in a position to show the correspondence between kernel contraction and base dependence (§4.1). Next, we will show a more specific correspondence between saturated kernel contraction and strong base dependence $(\S 4.2)$. Then we will make the latter correspondence yet more specific by factoring in composite contraction and composite dependence ( $\S 4.3)$.

We start by the following handy lemmas (connecting belief change and base dependence) that simplify some of the proofs for this section.

Lemma 30. In the presence of Def- $B$, Cond-ID ${ }^{B}$ and contribution, the following is equivalent to Cond $\stackrel{\div}{\div}: \quad \beta \in B \div \alpha$ iff $\beta \in B$ and $\alpha \not \rightarrow \beta$.

Lemma 31. Given relations $\leadsto$ and $\div$ for base $B$ such that Cond $\overline{\div}$ holds and contribution is satisfied, it also holds that: $\quad$ If $\beta \in B \div \alpha$ then $\alpha \neq \beta$. 


\subsection{Kernel Contraction and Base Dependence}

We now show the correspondence between kernel contraction and base dependence.

\subsubsection{From Base Dependence to Base Contraction}

To construct a contraction operator $\div$, assume the following are present: a base dependence relation $\leadsto$ (Definition 19), the tautologies in the belief base Taut $(B)$ (Definition 15), and Cond $\stackrel{\div}{\circ}$ We do not need to assume that $B$ is provided because, as discussed, we can obtain $B$ using $\leadsto$ and Taut $(B)$ via (5):

$B=B \lesssim=\{\beta \mid \beta \in \operatorname{Taut}(B)$ or $\alpha \lesssim \beta$ for some $\alpha\}$.

Theorem 32 states that the contraction operator $\div$ obtained from $\leadsto$ is indeed a kernel contraction.

Theorem 32 (Base Dependence to Base Contraction). Given relations $\leadsto$ and $\div$ for belief base $B$ such that Cond $\div$ holds, if $\leadsto$ is a base dependence, then $\div$ is a kernel contraction.

\subsubsection{From Base Contraction to Base Dependence}

This subsection shows how to obtain a base dependence $\leadsto$ relation given a kernel contraction operator $\div$. Assume the following are present: a kernel contraction operator $\div$, and Cond $\leadsto$. Theorem 33 states that, given the above assumptions, all axioms of base dependence $\leadsto$ relation are satisfied.

Theorem 33 (Base Contraction to Base Dependence). Given relations $\leadsto$ and $\div$ for belief base $B$ such that Cond $\leadsto$ holds, if $\div$ is a kernel contraction, then $\leadsto$ is a base dependence.

\subsubsection{Axiomatic Characterization}

We now need an axiomatic characterization theorem. We also adopt the FH assumption in Remark 10 of $\S 2.5$, which means that inclusion needs to be assumed in the characterization theorem. The rationale for this assumption is as follows. When constructing the $\leftrightharpoons$ relation using a contraction function via Cond $\leadsto$, the set of all $\beta$ such that $\alpha \leadsto \beta$ is identical to those $\beta \in B$ and $\beta \notin B \div \alpha$, or using set difference notation $\beta \in B \backslash(B \div \alpha)$. We know as a matter of fact that $B \div \alpha \subseteq B$ holds because, by Definition 2, any contraction operator satisfies inclusion. However, even if, for the sake of argument, $\div$ did not satisfy inclusion and there were some statements in $B \div \alpha$ that were not in $B$, such statements would have been lost in the set difference $\beta \in B \backslash(B \div \alpha)$. In turn, this means that to use $\leadsto$ to construct a contraction $\div$ via Cond $\leadsto$, we do not have enough information to prove or disprove inclusion. Instead, we have to assume that $\div$ already satisfies inclusion. Since all contraction functions satisfy inclusion (Definition 2), this assumption is not a serious loss of generality.

Theorem 34 (Characterization). Let the relations $\leadsto$ and $\div$ for belief base B be such that $\div$ satisfies inclusion, and that Cond $\leadsto$ holds. Then, $\div$ is a kernel contraction if and only if $\leadsto$ is a base dependence.

To prove this characterization theorem, we show that in presence of inclusion, Cond $\leadsto$ entails Cond $\overline{\div}$. Thus, assuming inclusion and Cond $\leadsto$, based on Theorems 32 and 33, kernel contraction and base dependence are logically equivalent. 


\subsection{Saturated Kernel Contraction and Strong Base Dependence}

As discussed in $\S 2.3$, saturated kernel contraction is a subclass of kernel contraction that is a reversible generalization of basic AGM contraction. That is, saturated kernel contraction is a base contraction, and when the belief base is in fact a belief set, it is equivalent to AGM partial meet contraction (Hansson, 1994).

In this section, we show that indeed saturated kernel contraction and strong base dependence have a correspondence.

\subsubsection{From Base Dependence to Base Contraction}

To construct a contraction operator $\div$, assume the following are present: a strong base dependence relation $\leadsto$ (Definition 20), the tautologies in the belief base Taut $(B)$ (Definition 15), and Cond $\overline{\div}$. Again as in $\S 4.1 .1, B$ can be obtained using $\leadsto$ and Taut $(B)$ via $(5)$.

Theorem 35 states that the contraction operator $\div$ obtained from $\leadsto$ is indeed a saturated kernel contraction.

Theorem 35 (Base Dependence to Base Contraction). Given relations $\leadsto$ and $\div$ for belief base $B$ such that Cond $\div$ holds, if $\leadsto$ is a strong base dependence, then $\div$ is a saturated kernel contraction.

\subsubsection{From Base Contraction to Base Dependence}

We assume the following are present: a saturated kernel contraction operator $\div$, and the Cond $\doteqdot$. Theorem 36 states that, given the above assumptions, all axioms of strong base dependence $\leadsto$ relation are satisfied.

Theorem 36 (Base Contraction to Base Dependence). Given relations $\leadsto$ and $\div$ for belief base $B$ such that Cond $\leadsto$ holds, if $\div$ is a saturated kernel contraction, then $\leadsto$ is a strong base dependence.

\subsubsection{AXIOMATIC CHARACTERIZATION}

As in $\S 4.1 .3$, and in common with FH (Remark 10), we assume that the given contraction operator satisfies inclusion in order to provide an axiomatic characterization theorem.

Theorem 37 (Characterization). Let the relations $\leadsto$ and $\div$ for belief base B be such that $\div$ satisfies inclusion and that Cond $\leadsto$ holds. Then, $\div$ is a saturated kernel contraction if and only if $\leadsto$ is a strong base dependence.

To prove this characterization theorem, we show that in presence of inclusion, Cond $\leadsto$ entails

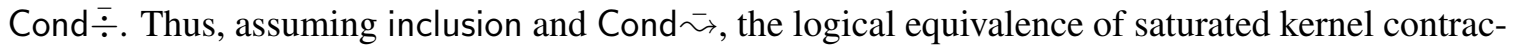
tion and strong base dependence follow from Theorems 35 and 36.

\subsection{Factoring in Composite Dependence}

We have seen Keynes's Conjunction Criterion for Dependence (CCD) and Gärdenfors's Conjunction Criterion for Independence $(\mathrm{CCl})$, and the respective dependence axioms $\mathrm{CCD}^{l}$ and $\mathrm{CCl}^{l}$. Likewise, we have:

If $\alpha \approx \delta$ and $\beta \approx \delta$ then $\alpha \wedge \beta \approx \delta$.

$\left(\mathrm{CCD}^{B}\right)$

If $\alpha \wedge \beta \leadsto \delta$ then $\alpha \leftrightharpoons \delta$ or $\beta \leadsto \delta$.

$\left(\mathrm{CCl}^{B}\right)$ 
$\mathrm{CCD}$ and $\mathrm{CCl}$ state intuitions regarding dependence on conjunctions in the form of conditional statements. One wonders whether it is possible to strengthen these postulates to exactly capture conjunctive dependence. Such a statement would have to capture different cases. That is, for any reasonable dependence relation, at least one of the following cases hold. The set of formulas that depend on $\alpha \wedge \beta$ is the same as at least one of the following:

Case 1: the set of those that depend on $\alpha$

Case 2: the set of those that depend on $\beta$

Case 3: the set of those that depend on $\alpha$ or depend on $\beta$.

These cases can be rephrased as follows:

Either $\{\delta \mid \alpha \wedge \beta \approx \delta\}=\{\delta \mid \alpha \approx \delta\}$, or

$$
\begin{aligned}
& \{\delta \mid \alpha \wedge \beta \leadsto \delta\}=\{\delta \mid \beta \leadsto \delta\}, \text { or } \\
& \{\delta \mid \alpha \wedge \beta \leadsto \delta\}=\{\delta \mid \alpha \leadsto \delta\} \cup\{\delta \mid \beta \leftrightharpoons \delta\}
\end{aligned}
$$

or equivalently,

Either $[\alpha \wedge \beta \approx \delta$ iff $\alpha \approx \delta]$, or

$$
\begin{aligned}
& {[\alpha \wedge \beta \leadsto \delta \text { iff } \beta \leadsto \delta], \text { or }} \\
& {[\alpha \wedge \beta \approx \delta \text { iff } \alpha \leftrightharpoons \delta \text { or } \beta \leftrightharpoons \delta] .}
\end{aligned}
$$

$\mathrm{CCDF}^{B}$ is a formalization of the intuition expressed in the three cases above, which we restate more concisely as follows, calling it the Conjunction Criterion of Dependence Factoring,

The set of all formulas that depend on $\alpha \wedge \beta$ is the same as the set of all formulas that depend on $\alpha$, or all formulas that depend on $\beta$, or all formulas that depend on $\alpha$ or on $\beta$.

Indeed, CCDF may be considered as a third principle for dependence of conjunctions in addition to Keynes's CCD and Gärdenfors's CCl.

As a side note, although it seems that the third clause of $\mathrm{CCDF}^{B}$ should be redundant, in light of the first two, in fact it isn't.

Example 38. Assume $a, b, c, d$ and $e$ are formulas and $\lesssim$ is a relation such that all the following hold:

$$
\begin{aligned}
& a \wedge b \leadsto c, \quad a \leadsto c, \quad b \neq_{\star} c, \\
& a \wedge b \leadsto d, \quad a \not d, \quad b \leadsto d, \\
& a \wedge b \leadsto e, \quad a \leadsto e, \quad b \leadsto e .
\end{aligned}
$$

Clearly, $\leadsto$ violates the first two clauses of $\mathrm{CCDF}^{B}$, but not the third one. This may be easier to see using (6), which is equivalent to $\operatorname{CCDF}^{B}$. Note that $\{\delta \mid a \wedge b \leftrightharpoons \delta\}=\{c, d, e\},\{\delta \mid a \leftrightharpoons \delta\}=\{c, e\}$ and $\{\delta \mid b \leftrightharpoons \delta\}=\{d, e\}$, which satisfy the third clause of (6) but not the first two. 
As a second note, $\mathrm{CCDF}^{B}$ is only one way of formalizing CCDF, using a base dependence relation; of course, it can also be formalized using FH's dependence relation as shown below, which we call $\mathrm{CCDF}^{l}$ :

Either $[\alpha \wedge \beta \leadsto \delta$ iff $\alpha \leadsto \delta]$, or

$$
\begin{aligned}
& {[\alpha \wedge \beta \leadsto \delta \text { iff } \beta \leadsto \delta], \text { or }} \\
& {[\alpha \wedge \beta \leadsto \delta \text { iff } \alpha \leadsto \delta \text { or } \beta \leadsto \delta] .}
\end{aligned}
$$

Finally, an important observation here is that $\mathrm{CCDF}^{B}$ is a more specific criterion than $\mathrm{CCD}^{B}$ and $\mathrm{CCl}^{B}$, and it implies both of them.

Theorem 39. If a relation $\leadsto$ satisfies $\mathrm{CCDF}^{B}$, then it also satisfies both $\mathrm{CCD}^{B}$ and $\mathrm{CCI}^{B}$.

Notice that although Theorem 39 is stated in terms of base dependence $\leadsto$, it does not have to be. Indeed, the theorem (and its proof) may straightforwardly be restated in terms of CCDF, which implies both CCD and CCI. As such, the dependence version of CCDF, i.e. CCDFl, also implies both $\mathrm{FH}^{\prime} \mathrm{CCD}^{l}$ and $\mathrm{CCl}^{l}$.

The following are all the conjunction criteria and related axioms we have discussed:

\begin{tabular}{|c|c|c|}
\hline Criterion & Dependence & Base Dependence \\
\hline $\mathrm{CCD}$ (Keynes) & $\mathrm{CCD}^{l}(\mathrm{FH})$ & $\mathrm{CCD}^{B}$ \\
$\mathrm{CCl}$ (Gärdenfors) & $\mathrm{CCl}^{l}(\mathrm{FH})$ & $\mathrm{CCl}^{B}$ \\
$\mathrm{CCDF}$ & $\mathrm{CCDF}^{l}$ & $\mathrm{CCDF}^{B}$ \\
\hline
\end{tabular}

We may now state an axiomatic characterization and its associated theorems, with the addition of the corresponding conjunction criteria as follows.

Theorem 40 (Base Dependence to Base Contraction). Given relations $\leadsto$ and $\div$ for belief base $B$ such that Cond $\div$ holds, if $\leadsto$ is a strong base dependence that satisfies $\mathrm{CCDF}^{B}$, then $\div$ is a saturated kernel contraction that satisfies conjunctive factoring.

Theorem 41 (Base Contraction to Base Dependence). Given relations $\approx$ and $\div$ for belief base $B$ such that Cond $\leadsto$ holds, if $\div$ is a saturated kernel contraction that satisfies conjunctive factoring, then $\leadsto$ is a strong base dependence that satisfies $\mathrm{CCDF}^{B}$.

Theorem 42 (Main Characterization). Let the relations $\leadsto$ and $\div$ for belief base $B$ be such that $\div$ satisfies inclusion, $B \div \alpha \subseteq B$, and that Cond $\leadsto$ holds. Then, $\div$ is a saturated kernel contraction that satisfies conjunctive factoring if and only if $\leadsto$ is a strong base dependence that satisfies $\mathrm{CCDF}^{B}$.

\section{Strong Base Dependence as a Reversible Generalization of Dependence}

Lastly, we show that base dependence is a reversible generalization of FH's dependence.

Theorem 43 (Dependence Generalization). Let relations $\leadsto, \sim$ and $\div$ for belief base $B$ be such that Cond $\leadsto$ and Cond $\leadsto$ hold respectively and inclusion is satisfied. In the case where $B$ is logically closed, $B=\mathrm{Cn}(B)$,

(1) the following are logically equivalent: 


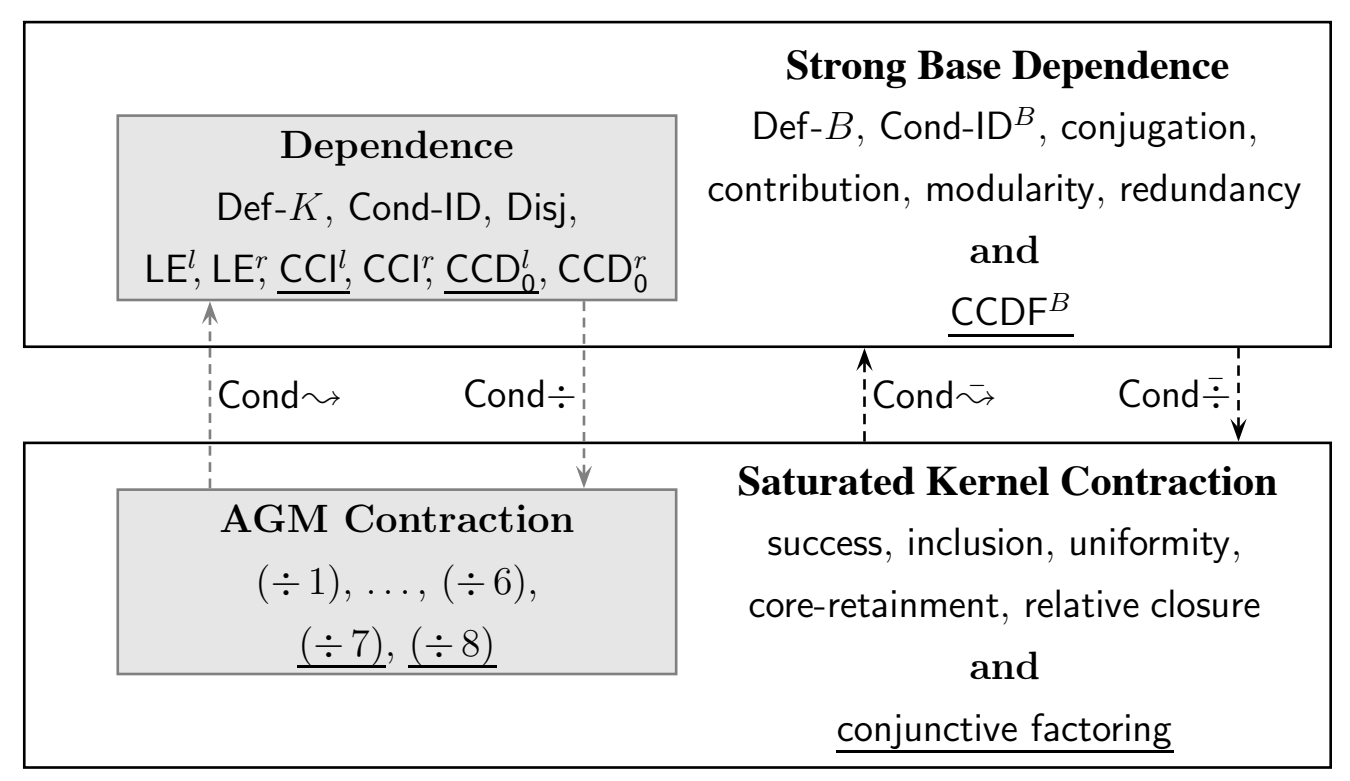

Figure 3: Belief Change and Base Dependence (omitting the underlined axioms results in a weaker characterization).
a) $\doteqdot$ is a strong base dependence
b) $\sim$ is a dependence that satisfies Def- $K$, Cond-ID, Disj, LE, $\mathrm{LE}^{r}, \mathrm{CCl}^{r}$ and $\mathrm{CCD}_{0}^{r}$
c) $\div$ is a saturated kernel contraction
d) $\div$ is a basic AGM contraction function, which satisfies $(\div 1)-(\div 6)$
(2) if any one of 1.a-1.d above holds, then $\leadsto$ reduces to $\leadsto$ :
$\alpha \leadsto \beta$ iff $\alpha \leadsto \beta$.

In the theorem above, the given list of axioms for the dependence relation $\leadsto$ does not include all the 9 axioms that FH have put forward for dependence. Rather, it is a subset of their axioms that corresponds to basic AGM contraction function satisfying $(\div 1)-(\div 6)$. To be able to account for $(\div 7)$ and $(\div 8)$ as well, we need to use all of their 9 axioms that correspond to full AGM contraction function, $(\div 1)-(\div 8)$. This in turn means that we also need a base contraction that, for closed sets, is equivalent to full AGM contraction. This can be shown to be the case for saturated kernel contraction that also satisfies conjunctive factoring.

Lemma 44. In the special case where base $B$ is logically closed, an operator $\div$ on $B$ is an $A G M$ contraction satisfying $(\div 1)-(\div 6),(\div 7)$ and $(\div 8)$ if and only if $\div$ is a saturated kernel contraction that satisfies conjunctive factoring.

Now everything is in place to extend the formalism for a base dependence relation $\leadsto$ that also satisfies $\mathrm{CCDF}^{B}$. Satisfying $\mathrm{CCDF}^{B}$ allows $\leadsto$ to meet both $\mathrm{CCD}$ and $\mathrm{CCl}$. 
Theorem 45 (Dependence Generalization with Conjunction). Let relations $\leadsto, \leadsto$ and $\div$ for belief base $B$ be such that Cond $\sim$ and Cond $\sim$ hold respectively and inclusion is satisfied. In the case where $B$ is logically closed, $B=\mathrm{Cn}(B)$,

(1) the following are logically equivalent:

a) $\leadsto$ is a strong base dependence that satisfies $\mathrm{CCDF}^{B}$

b) $\leadsto$ is a dependence, which satisfies Def- $K$, Cond-ID, Disj, $\mathrm{LE}^{l}, \mathrm{LE}^{r}, \mathrm{CCl}^{l}, \mathrm{CCl}^{r}, \mathrm{CCD}_{0}^{l}$ and $\mathrm{CCD}_{0}^{r}$

c) $\div$ is a saturated kernel contraction that satisfies conjunctive factoring

d) $\div$ is an AGM contraction function, which satisfies $(\div 1)-(\div 6),(\div 7)$ and $(\div 8)$

(2) if any one of 1.a-1.d above holds, then $\leadsto$ reduces to $\leadsto$ :

$\alpha \leadsto \beta$ iff $\alpha \leadsto \beta$.

The diagram in Figure 3 captures the key results from Theorems 35-45.

\section{Discussion and Related Work}

Linking belief change and dependence can be of great value because, for example, it can narrow the number of formulas that need to be considered during a belief change operation. This, in turn, can greatly improve the performance of the operation. Gärdenfors' preservation criterion suggests a particularly interesting way of establishing this link. Work of great theoretical value based on Gärdenfors' preservation criterion is that of FH that focuses on the relationship between dependence and AGM theory contraction.

\subsection{Discussion}

In the present work, we take a natural next step of finding a similar connection between dependence and belief base contraction that can have important practical consequences. We call such a dependence relation base dependence. Since belief bases (which can be closed or non-closed) are a generalization of belief sets, it would be nice if their corresponding dependence relation, base dependence, also turns out to be a generalization of FH's dependence relation.

In this work, we establish such a connection between belief base contraction and base dependence. That is, we provide an axiomatization of base dependence, and establish its relation to belief base contraction. Similar to the set of axioms suggested by FH, the base dependence axioms are also meant to capture the dependence among formulas. However, for base dependence the formulas are from the belief base, which may or may not be closed. Thus base dependence generalizes dependence.

More interestingly, base dependence turns out to be a reversible generalization of dependence. That is, we prove that in the special case that a belief base is deductively closed (i.e., it is a belief set), the base dependence relation reduces to the original FH's dependence relation.

What sets apart FH's approach from that of other authors (see the Discussion and Related Work below) is its integration into the AGM model, being closely intertwined with AGM contraction. This in turn means that their work provides a theoretical limit for other approaches trying to capture or approximate concepts of relevance and dependence in the context of belief change. By generalizing their work, our approach inherits this useful property for both belief bases and belief sets. 
On a separate note, another interesting characteristic of FH's axioms for dependence is that some of them are based on intuitions stated by previous authors working on the notion of relevance and dependence such as Keynes (1921) and Gärdenfors (1978, 1990). More specifically, their dependence axiomatization $(\S 2.5)$ meets both Keynes's conjunction criterion for dependence, CCD, and Gärdenfors's conjunction criterion for independence, $\mathrm{CCl}$.

Not only does our base dependence generalization preserves this characteristic of dependence, but we also go one step further and provide a more specific intuition called conjunction criterion of dependence factoring, CCDF, that encompasses both Keynes's CCD and Gärdenfors's CCI intuitions.

In summary, the new contributions in this work include:

- An axiomatization of base dependence relation for belief base formulas.

- Characterization theorems to construct base dependence via belief base contraction and vice versa, similar to epistemic entrenchment in AGM.

- Proving that the new base dependence relation is a reversible generalization of FH's dependence relation.

- Generalizing the dependence relation and showing how base dependence preserves some of the most interesting properties of dependence, particularly, Keynes's conjunction criterion of dependence, CCD, and Gärdenfors's conjunction criterion of independence, $\mathrm{CCl}$.

- Introducing the conjunction criterion of dependence factoring, CCDF, which is more specific than $\mathrm{CCD}$ and $\mathrm{CCl}$, and entails both of them.

\subsection{Related Work}

There has been extensive research regarding relevance both with respect to belief change and beyond. The present work, in common with FH, doesn't directly provide a theory of relevance. Rather the goal is to determine the notion of relevance (or, dependence) induced by a belief base contraction operator. In the same way, FH examine what the counterpart is for AGM contraction in terms of dependence; thus their dependence relation is a counterpart to a contraction function in the same way that an epistemic entrenchment relation on formulas exactly characterises a contraction function.

Hansson and Wassermann (2002) classify work that addresses the concepts of relevance and dependence of formulas into two groups. First, some authors capture relevance/dependence of formulas through syntactic means such as variable sharing and language splitting (including Chopra \& Parikh, 2000; Falappa, García, Kern-Isberner, \& Simari, 2011; Ismail \& Kasrin, 2010; Ji, Qi, \& Haase, 2008; Kourousias \& Makinson, 2007; Makinson \& Kourousias, 2006; Makinson, 2007; Parikh, 1999; Perrussel, Marchi, \& Zhang, 2011; Suntisrivaraporn, Qi, Ji, \& Haase, 2008; Wu, Zhang, \& Zhang, 2011). Second, some authors focus on inferential dependency of formulas or, in other words, how some formulas deductively contribute to the inference of other formulas. Examples of this approach include the works of Cuenca Grau, Halaschek-Wiener, and Kazakov (2007), Delgrande and Pelletier (1998), Fariñas del Cerro and Herzig (1996), Hansson and Wassermann (2002), as well as the present work. Typically, syntactic approaches are simpler and computationally more efficient compared to inferential approaches. However, the latter usually provide a more precise and tighter definition of relevance and dependence than syntactic approaches. 


\subsubsection{COMPARISON WITH FH'S APPROACH}

The work most related to our study is that of Fariñas del Cerro and Herzig in Belief Change and Dependence (1996). Here we analyze some high-level properties that set FH work apart from other studies, which are also inherited in our generalization of their work.

One comment some may make on FH's approach in studying the notion of dependence in the context of belief change is the following. An expected high level goal of such a study is to use dependence or relevance to reduce the number of candidate belief statements that can potentially be affected by a particular change. This should significantly improve tractability of belief change operations. On the other hand, a dependence relation in their work is constructed using a given AGM contraction operator. Thus, their way of constructing dependence relations may seem to some as a deviation from that original goal. There appears to be a circularity: dependence was meant to help with the process of belief change operations, but now its construction is based on such operations.

Nevertheless, there are quite a few important and sometimes unique benefits to their approach. First, even though their dependence relation is constructed based on AGM contraction, their axiomatization of dependence (as well as ours) is largely separate from the AGM concepts. Indeed, as seen in $\S 3.3$, many of the postulates in FH's axiomatization are based on concepts introduced even before the AGM model was developed, for example Keynes' CCD and Gärdenfors' CCI.

Moreover, construction of a dependence relation based on AGM contraction has an important implication: it provides the most theoretically-precise definition of dependence in the context of belief change. Indeed, this deep integration with the theory of belief change is what sets FH's work and our work apart from other related work. More specifically, the notions of dependence and belief change are connected as two sides of the same coin in all the correspondences that FH and we have demonstrated, namely, Belief Change and Dependence ( $\{2.5)$, Kernel Contraction and Base Dependence ( $(4.1)$, and Saturated Kernel Contraction and Strong Base Dependence ( $(4.2)$. This provides a theoretically sound definition of dependence in the context of belief change-a theoretical benchmark that, for example, other approximating approaches can be compared against. To help clarify this point, in the following we look at another similar relationship for comparison.

\subsubsection{COMPARISON WITH SYNTACTIC APPROACHES}

To demonstrate how our approach can be used as a theoretical benchmark for approximating approaches to relevance, in the following example we consider how (in)compatible a given approximate dependence relation is with saturated kernel contraction. Riani and Wassermann (2004) (see also Wassermann, 1999) provide the syntactic relevance relation $R$ as follows: $R(\alpha, \beta)$ if and only if the formulas $\alpha$ and $\beta$ share an atom. Simply put, $R$ just amounts to variable sharing. Then, for instance, to speed up belief change operations, one may want to find out to what extent $R$ is compatible with belief change. In the following example, we consider only one base dependence axiom redundancy (see $\S 3.3$ and $\S 4.2$ ), and we denote $R(\alpha, \beta)$ with $\alpha \overline{\sim_{R}} \beta$.

Example 46. Assume that $B=\{p, q, p \vee q\}$, and that $\bar{\sim}_{R}$ is a relation constructed based on variable sharing such that, for example, $p \overline{\sim_{R}} p, p \overline{\nexists_{R}} q$ and $p \overline{\sim_{R}}(p \vee q)$ hold. We show that $\overline{\sim_{R}}$ violates redundancy. Let $B^{\prime}=\{q\}$. Clearly $B^{\prime} \subseteq B$ and $p \vee q \in \operatorname{Cn}\left(B^{\prime}\right)$, and, as stated above, $p \bar{\sim}_{R}(p \vee q)$. Thus, by redundancy, $p{\overline{\sim_{R}}}_{\delta}$ for some $\delta \in B^{\prime}$. Since $B^{\prime}=\{q\}, \delta$ can only be $q$, so $p \bar{\sim}_{R} q$. This contradicts $p \Varangle_{R} q$. 
Now, let us relax $R$ (as Riani and Wassermann do), and even though $p$ and $q$ do not share any atoms, we allow $R(p, q)$, or $p \bar{\sim}_{R} q$, because after all $R(p, p \vee q)$ and $R(p \vee q, q)$. Then, since in Example 46, we have $p \overline{\sim_{R}} q$, it does not violate redundancy any more. That is, we can study to what extent a relation $R$ is compatible with belief change without referring to belief change axioms at all. It is more convenient to conduct a study about dependence relations using only dependence axioms.

\subsubsection{COMPARISON WITH INFERENTIAL APPROACHES}

An example of studies considering inferential dependency (as opposed to considering syntactic means) is that of Hansson and Wassermann (2002). They "consider relevant to a formula $\alpha$ the formulas that appear in a minimal derivation of $\alpha$ or its negation." They use belief bases and interestingly they also use kernels to come up with minimal derivations for formulas. Thus, there is a significant amount of common ground between their study and base dependence, which corresponds to saturated kernel contraction (which is also based on kernels). Nevertheless, their formalism and concept of dependence (relevance) differs from that of FH's and from ours in some important ways. For instance, for them, anything that depends on $\alpha$ also depends on $\neg \alpha$. In contrast, $\alpha$ and $\neg \alpha$ can never depend nor base-depend on each other: $\alpha \not \neg \neg \alpha$ and $\alpha \not \neg \neg \alpha$ by Disj and Disj ${ }^{B}$, respectively. This turns out to be important for mutuality of dependence and belief change or similarly base dependence and belief change. More specifically, they can construct contraction operators from given dependence relations (similar to Cond $\div$ or Cond $\overline{\div}$ ), but they cannot construct dependence relations if given contraction operators (lacking anything similar to Cond $\sim$ or Cond $\leadsto$ ).

\subsection{Future Work}

There are a number of future research paths from this study. Here we provide some examples of open questions and possible research directions.

\subsubsection{BASE DEPENDENCE AND Formulas of THE BASE}

As discussed in $\S 3$, a base dependence relation $\leadsto$ in this study guarantees statement (4):

If $\alpha \leadsto \beta$ then $\beta \in B$.

This was not needed to be explicitly stated as an axiom because it is implied by the set of axioms offered for the base dependence relationship.

There remain two other alternative approaches which could be explored in other studies, albeit not being as general as the above approach. First, instead of requiring $\beta \in B$, require that $\alpha \in B$.

If $\alpha \leadsto \beta$ then $\alpha \in B$.

This alternative can be useful when we are interested on the effect of changing the base on other statements.

Another alternative requires both $\alpha, \beta \in B$.

If $\alpha \leadsto \beta$ then $\alpha \in B$ and $\beta \in B$.

This alternative may be particularly useful in a study of redundancy in the base. That is, exploring how removal of statements from the base requires removal of other statements in the base which can happen in the presence of redundant statements. 


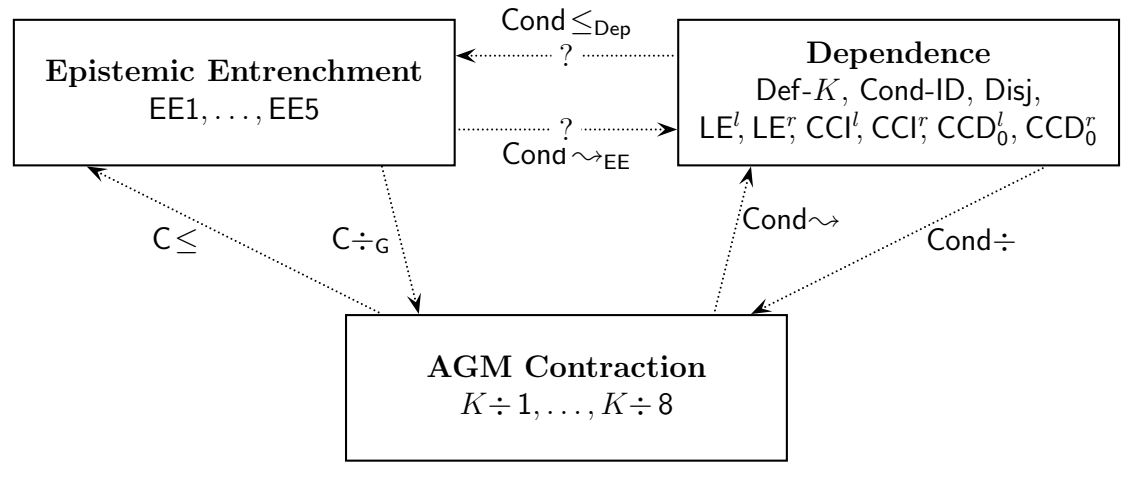

Figure 4: Open Problem: Provide the conditions Cond $\leadsto$ EE and Cond $\leq_{\text {Dep }}$ and representation theorems to directly connect Dependence to Epistemic Entrenchment.

\subsubsection{Direct Proof of Base Dependence Generalizing Dependence}

In $\S 5$, we showed indirectly that the set of base dependence relations is a superset of the set of $d e$ pendence relations. In principle, this could be shown directly using the axioms of base dependence and dependence.

For some of the base dependence axioms it is straightforward to see their connection with their counterpart dependence axioms; e.g. Disj ${ }^{B}$, Cond-ID ${ }^{B}$ and Def- $B$. For some other axioms such as modularity and redundancy, it seems to be more delicate to prove.

Furthermore, the current proof of Theorem 43 is “indirect" also because it uses Hansson's (1994) Theorem 6 to establish the relationship between partial meet contraction and base dependence (when dealing with closed sets). It should be possible to study this relationship more directly. One may wish to pursue this possibility considering the following "variation" of the contribution axiom:

If $\alpha \leadsto \beta$ then $\alpha \notin \mathrm{Cn}\left(B^{\prime}\right)$ and $\alpha \in \mathrm{Cn}\left(B^{\prime} \cup\{\beta\}\right)$

for some $B^{\prime}$ such that $\{\delta \mid \delta \in B$ and $\alpha \not \Varangle \delta\} \subseteq B^{\prime} \subseteq B$.

The conjecture that axiom (7) corresponds to relevance is intuitively appealing. If this conjecture is proved then it may help to show the relationship between base dependence and partial meet contraction more directly. ${ }^{7}$

\subsubsection{DEPENDENCE AND EPISTEMIC ENTRENCHMENT}

Both dependence and epistemic entrenchment are counterparts of AGM contraction. Thus, it is only natural to expect to find a strong and more direct connection between them. Interestingly, the condition $C \div \mathrm{G}$ "gives an explicit answer to which sentences are included in the contracted belief set, given the initial belief set and an ordering of epistemic entrenchment" (Gärdenfors, 2003, p. 19). Indeed, dependence is also concerned with specifying which sentences stay in the contracted belief set and which ones do not. This hypothetical relation is shown in Figure 4. The unknown conditions

7. We would like to thank one of our anonymous reviewers for suggesting axiom (7) and its possible relationship with relevance and partial meet contraction. 
Cond $\sim_{E E}$ and Cond $\leq_{\text {Dep }}$ can help to provide a more direct connection between dependence and epistemic entrenchment.

The topic in this subsection "Dependence and Epistemic Entrenchment" is perhaps more appropriate for the original FH's approach to dependence, but it also applies to the present work. As noted in the Related Work section ( $(6.2)$, there have been a number of proposals for dealing with relevance in belief change; such characterizations of relevance are often expressed via additional postulates (for example, see Axiom $\mathbf{P}$ in Parikh, 1999). It would be of interest then to examine the counterparts of such postulates as reflected in additional constraints on dependency relations.

\subsubsection{BASE DEPENDENCE AND ENSCONCEMENT}

On the one hand, there is a potential relationship between dependence and epistemic entrenchment as shown in Figure 4. On the other hand, it is known that an epistemic entrenchment formalism for belief bases is not possible. "Probably the most successful application to belief bases of the ideas behind entrenchment is the theory of ensconcement relations that has been developed by MaryAnne Williams (1994)," Hansson (1999, p. 103) states. Therefore, one interesting topic to explore is to find the relationship between base dependence and ensconcement.

\section{Conclusion}

Gärdenfors' preservation criterion suggests a particularly interesting way of establishing a link between belief change and dependence. Such dependence relations can in turn be used as a theoretical benchmark against which to evaluate other approximate dependence or relevance relations. We have built on GPC and provided the most general formulation of it currently available (to the best of our knowledge). Basically, there are three corresponding pairs: (1a) AGM contraction is a subclass of (1b) saturated kernel contraction, which is a subclass of (1c) kernel contraction; likewise, (2a) FH's dependence is a subclass of (2b) strong base dependence, which is a subclass of (2c) base dependence. Our formalism connects the most general pair from each group, (1c) and (2c): kernel contraction and base dependence, as well as an important special case connecting pair (1b) and ( $2 \mathrm{~b}$ ): saturated kernel contraction and strong base dependence. In the case when a belief base is closed, the generalized dependence, strong base dependence, is equivalent to the original FH dependence relation. While generalizing FH's work, we preserve some of the important characteristics of their study such as Keynes's conjunction criterion for dependence (CCD) and Gärdenfors's conjunction criterion for independence $(\mathrm{CCl})$. Additionally, we provide a more specific intuition called conjunction criterion of dependence factoring, CCDF, that encompasses both Keynes's CCD and Gärdenfors's $\mathrm{CCl}$ intuitions.

We have explored different conditions to construct base dependence relations using belief contraction operators. In doing so we have fully expanded the usage of an existing condition to construct base dependence, Cond $\sim$. We have also come up with new conditions, strong base dependence Cond $\hat{\sim}$ and weak base dependence Cond $\check{\sim}$, and described their various relations to one another.

We also provide the means to study redundancy in light of weak base dependence. The fact that weak base dependence captures redundancy may be exploited for different purposes. For example, one may use weak base dependence to distinguish between redundant and informative formulas in a belief base. 


\section{Appendix A. Proofs}

All theorems and their proofs are gathered in this appendix so that it is easier to review them, and to help avoid clutter.

\section{A.1 Proofs for $\S 3.3$ : Basic Postulates of Base Dependence}

Theorem 17. If a relation $\leadsto$ satisfies conjugation, then it also satisfies $\mathrm{LE}^{B}$.

\begin{tabular}{|c|c|c|}
\hline \multicolumn{3}{|c|}{ Proof. } \\
\hline 1 & $\begin{array}{l}\text { If } \alpha \in \operatorname{Cn}\left(B^{\prime}\right) \text { iff } \beta \in \operatorname{Cn}\left(B^{\prime}\right) \text { for all } B^{\prime} \subseteq B \\
\text { then } \alpha \leadsto \delta \text { iff } \beta \leadsto \delta\end{array}$ & conjugation \\
\hline 2 & $\alpha \leftrightarrow \beta$ & Assumption \\
\hline 3 & $\alpha \rightarrow \beta$ & 2 \\
\hline 4 & $\beta \rightarrow \alpha$ & 2 \\
\hline 5 & If $\alpha \in \operatorname{Cn}(C)$ then $\beta \in \operatorname{Cn}(C)$ for all $C$ & 3 , supraclassicality for $\mathrm{Cn}$ \\
\hline 6 & If $\beta \in \operatorname{Cn}(C)$ then $\alpha \in \operatorname{Cn}(C)$ for all $C$ & 4, supraclassicality for $\mathrm{Cn}$ \\
\hline 7 & $\alpha \in \operatorname{Cn}(C)$ iff $\beta \in \operatorname{Cn}(C)$ for all $C$ & 5,6 \\
\hline 8 & $\alpha \leadsto \delta$ iff $\beta \leadsto \delta$ & 1,7 \\
\hline 9 & If $\alpha \leftrightarrow \beta$ then $\alpha \leadsto \delta$ iff $\beta \leadsto \delta$ & 2,8 \\
\hline 10 & If $\vdash \alpha \leftrightarrow \beta$ and $\alpha \leadsto \delta$ then $\beta \leadsto \delta$ & 9; $\mathrm{LE}^{B}$ derived \\
\hline
\end{tabular}

Theorem 18. If a relation $\leftrightharpoons$ satisfies contribution, then it also satisfies Disj $^{B}$.

\begin{tabular}{|c|c|c|}
\hline \multicolumn{3}{|c|}{ Proof. } \\
\hline 1 & $\begin{array}{l}\text { If } \alpha \leadsto \beta \text { then } \\
\quad \alpha \notin \operatorname{Cn}\left(B^{\prime}\right) \text { and } \alpha \in \operatorname{Cn}\left(B^{\prime} \cup\{\beta\}\right) \text { for some } B^{\prime} \subseteq B\end{array}$ & contribution \\
\hline 2 & $\begin{array}{l}\text { If } \alpha \in \operatorname{Cn}\left(B^{\prime}\right) \text { or } \alpha \notin \operatorname{Cn}\left(B^{\prime} \cup\{\beta\}\right) \text { for all } B^{\prime} \subseteq B \\
\text { then } \alpha \not \Varangle \beta\end{array}$ & 1 (contrapositive) \\
\hline 3 & $\vdash \alpha \vee \beta$ & Assumption \\
\hline 4 & $\alpha \vee \beta \in \operatorname{Cn}(\emptyset)$ & 3 \\
\hline 5 & $\alpha \vee \beta \in \operatorname{Cn}(C)$ & 4 , monotony for $\mathrm{Cn}$ \\
\hline 6 & $\alpha \in \operatorname{Cn}(C \cup\{\beta\})$ & Assumption \\
\hline 7 & $(\beta \rightarrow \alpha) \in \operatorname{Cn}(C)$ & 6 , deduction for $\mathrm{Cn}$ \\
\hline 8 & $(\alpha \vee \neg \beta) \in \operatorname{Cn}(C)$ & 7, supraclassicality for $\mathrm{Cn}$ \\
\hline 9 & $((\alpha \vee \beta) \wedge(\alpha \vee \neg \beta)) \in \operatorname{Cn}(C)$ & 5,8 , supraclassicality for $\mathrm{Cn}$ \\
\hline 10 & (using the resolution rule) & 9, supraclassicality for $\mathrm{Cn}$ \\
\hline 11 & If $\alpha \in \operatorname{Cn}(C \cup\{\beta\})$ then $\alpha \in \operatorname{Cn}(C)$ & 6,10 \\
\hline 12 & {$\left[\alpha \in \mathrm{Cn}\left(B^{\prime} \cup\{\beta\}\right)\right.$ or $\left.\alpha \notin \operatorname{Cn}\left(B^{\prime} \cup\{\beta\}\right)\right]$ for all $B^{\prime} \subseteq B$} & Tautological truth \\
\hline 13 & {$\left[\alpha \in \operatorname{Cn}\left(B^{\prime}\right)\right.$ or $\left.\alpha \notin \operatorname{Cn}\left(B^{\prime} \cup\{\beta\}\right)\right]$ for all $B^{\prime} \subseteq B$} & 11,12 \\
\hline 14 & $\alpha \not \nrightarrow \beta$ & 2,13 \\
\hline 15 & If $\vdash \alpha \vee \beta$ then $\alpha \not \nrightarrow \beta$ & 3, 14; Disj $^{B}$ derived \\
\hline
\end{tabular}




\section{A.2 Proofs for $§ 3.5 .2$ : Connections Among (Base) Dependence Constructions}

Lemma 47. An operator $\div$ on base $B$ satisfies relative closure if and only if it satisfies the following:

If $\beta \in B$ then $\beta \in B \div \alpha$ iff $\beta \in \operatorname{Cn}(B \div \alpha)$.

Proof.

\begin{tabular}{l|l|l}
\hline 1 & $B \div \alpha \subseteq \operatorname{Cn}(B \div \alpha)$ & By inclusion for Cn \\
2 & If $\beta \in B \div \alpha$ then $\beta \in \operatorname{Cn}(B \div \alpha)$ & 1 \\
\hline 3 & $B \cap \operatorname{Cn}(B \div \alpha) \subseteq B \div \alpha$ & Assume relative closure \\
4 & If $\beta \in B$ and $\beta \in \operatorname{Cn}(B \div \alpha)$ then $\beta \in B \div \alpha$ & 3 \\
5 & If $\beta \in B$ then $[\beta \notin \operatorname{Cn}(B \div \alpha)$ or $\beta \in B \div \alpha]$ & 4 \\
6 & If $\beta \in B$ then $[$ if $\beta \in \operatorname{Cn}(B \div \alpha)$ then $\beta \in B \div \alpha]$ & 5 \\
7 & If $\beta \in B$ then $[\beta \in \operatorname{Cn}(B \div \alpha)$ iff $\beta \in B \div \alpha]$ & 2,$6 ;$ so (8) is derived \\
\hline 8 & Since lines 3 through 7 are logically equivalent, the reverse order also holds \\
\hline
\end{tabular}

Theorem 21. Given relations $\leadsto, \hat{\sim}, \check{\sim}$ and $\div$ for belief base $B$ such that Cond $\leadsto$, Cond $\hat{\sim}$ and

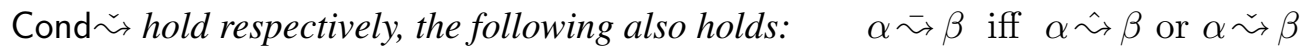

\begin{tabular}{|c|c|c|}
\hline \multicolumn{3}{|c|}{ Proof. } \\
\hline 1 & $B \div \alpha \subseteq \operatorname{Cn}(B \div \alpha)$ & By inclusion for $\mathrm{Cn}$ \\
\hline 2 & If $\beta \notin \operatorname{Cn}(B \div \alpha)$ then $\beta \notin B \div \alpha$ & 1 (by the set theory) \\
\hline 3 & $\alpha \leadsto \beta$ iff $\beta \in B$ and $\beta \notin B \div \alpha$ & Cond $\leadsto$ \\
\hline 4 & $\alpha \hat{\sim} \beta$ iff $\beta \in B$ and $\beta \notin \operatorname{Cn}(B \div \alpha)$ & Cond $\hat{\sim}$ \\
\hline 5 & $\begin{array}{l}\alpha \stackrel{\sim}{\sim} \beta \text { iff } \\
\quad \beta \in B \text { and } \beta \notin B \div \alpha \text { and } \beta \in \operatorname{Cn}(B \div \alpha)\end{array}$ & Cond $\sim$ \\
\hline 6 & $\alpha \leadsto \beta$ iff $\alpha \hat{\sim} \beta$ or $\alpha \leadsto \beta$ & Assumed to be verified \\
\hline 7 & $\begin{array}{l}{[\beta \in B \text { and } \beta \notin B \div \alpha] \text { iff }} \\
\quad[\beta \in B \text { and } \beta \notin \operatorname{Cn}(B \div \alpha)] \text { or } \\
\quad[\beta \in B \text { and } \beta \notin B \div \alpha \text { and } \beta \in \operatorname{Cn}(B \div \alpha)]\end{array}$ & $\begin{array}{l}3,4,5,6 \text { (substituting each term } \\
\text { in } 6 \text { with its equivalent) }\end{array}$ \\
\hline 8 & $\begin{array}{l}{[\beta \in B \text { and } \beta \notin B \div \alpha] \text { iff }} \\
{[\beta \in B \text { and }[\beta \notin B \div \alpha \text { and } \beta \notin \operatorname{Cn}(B \div \alpha)]] \text { or }} \\
{[\beta \in B \text { and } \beta \notin B \div \alpha \text { and } \beta \in \operatorname{Cn}(B \div \alpha)]}\end{array}$ & $\begin{array}{l}\text { 2, } 7 \text { (adding redundant conjunct } \\
\quad \beta \notin B \div \alpha \text { ) }\end{array}$ \\
\hline 9 & $\begin{array}{l}{[\beta \in B \text { and } \beta \notin B \div \alpha] \text { iff }} \\
\quad[[\beta \in B \text { and } \beta \notin B \div \alpha] \text { and } \beta \notin \operatorname{Cn}(B \div \alpha)] \text { or } \\
{[[\beta \in B \text { and } \beta \notin B \div \alpha] \text { and } \beta \in \operatorname{Cn}(B \div \alpha)]}\end{array}$ & 8 (regrouping conjuncts) \\
\hline 10 & $\begin{array}{l}{[\beta \in B \text { and } \beta \notin B \div \alpha] \text { iff }} \\
\quad[\beta \in B \text { and } \beta \notin B \div \alpha] \text { and } \\
{[\beta \notin \operatorname{Cn}(B \div \alpha) \text { or } \beta \in \operatorname{Cn}(B \div \alpha)]}\end{array}$ & 9 (factoring out the common term) \\
\hline 11 & $\begin{array}{l}{[\beta \in B \text { and } \beta \notin B \div \alpha] \text { iff }} \\
{[\beta \in B \text { and } \beta \notin B \div \alpha]}\end{array}$ & 10 (omitting tautological conjunct) \\
\hline 12 & $\top$ (i.e., reached a tautology) & 11 ; assumption 6 verified \\
\hline
\end{tabular}


Theorem 22. Given relations $\leadsto, \hat{\sim}$ and $\div$ for belief base B such that Cond $\leadsto$ and Cond $\hat{\sim}$ hold respectively and relative closure is satisfied, the base dependence relation $\leadsto$ and the strong base dependence relation $\hat{\sim}$ are equivalent: $\quad \alpha \leadsto \beta$ iff $\alpha \hat{\sim} \beta$.

\begin{tabular}{|c|c|c|}
\hline \multicolumn{3}{|c|}{ Proof. } \\
\hline$\overline{1}$ & $\alpha \leadsto \beta$ iff $\beta \in B$ and $\beta \notin B \div \alpha$ & Cond $\sim$ \\
\hline 2 & $\alpha \hat{\sim} \beta$ iff $\beta \in B$ and $\beta \notin \operatorname{Cn}(B \div \alpha)$ & Cond $\hat{\sim}$ \\
\hline 3 & $B \cap \operatorname{Cn}(B \div \alpha) \subseteq B \div \alpha$ & relative closure \\
\hline 4 & If $\beta \in B$ then $[\beta \in B \div \alpha$ iff $\beta \in \operatorname{Cn}(B \div \alpha)]$ & 3 and Lemma 47 \\
\hline 5 & If $\beta \in B$ then $[\beta \notin B \div \alpha$ iff $\beta \notin \operatorname{Cn}(B \div \alpha)]$ & 4 (negating both sides of iff) \\
\hline 6 & $\alpha \leadsto \beta$ iff $\alpha \hat{\sim} \beta$ & Assumed to be verified \\
\hline 7 & $\begin{array}{l}{[\beta \in B \text { and } \beta \notin B \div \alpha] \text { iff }} \\
{[\beta \in B \text { and } \beta \notin \operatorname{Cn}(B \div \alpha)]}\end{array}$ & $\begin{array}{l}1,2,6 \text { (substituting each term in } 6 \text { with } \\
\text { its equivalent) }\end{array}$ \\
\hline 8 & $\begin{array}{c}{[\beta \in B \text { and } \beta \notin B \div \alpha] \text { iff }} \\
{[\beta \in B \text { and } \beta \notin B \div \alpha]}\end{array}$ & $\begin{array}{l}5,7 \text { (substituting } \beta \notin \mathrm{Cn}(B \div \alpha \text { ) with } \\
\quad \beta \notin B \div \alpha \text { given the conjunct } \beta \in B \text { ) }\end{array}$ \\
\hline 9 & $\top$ (i.e., reached a tautology) & $8 ;$ assumption 6 verified \\
\hline
\end{tabular}

Theorem 23. Given relations $\leadsto, \leadsto$ and $\div$ for belief base B such that Cond $\leadsto$ and Cond $\leadsto$ hold respectively and relative closure is satisfied, we obtain: $\quad \alpha \leadsto \beta$ iff $\beta \in B$ and $\alpha \leadsto \beta$.

\begin{tabular}{|c|c|c|}
\hline \multicolumn{3}{|c|}{ Proof. } \\
\hline 1 & If $\beta \in B$ then $\beta \in \operatorname{Cn}(B)$ & By inclusion for $\mathrm{Cn}$ \\
\hline 2 & $\alpha \leadsto \beta$ iff $\beta \in B$ and $\beta \notin B \div \alpha$ & Cond $\leadsto$ \\
\hline 3 & $\alpha \leadsto \beta$ iff $\beta \in \operatorname{Cn}(B)$ and $\beta \notin \operatorname{Cn}(B \div \alpha)$ & Cond $\leadsto$ \\
\hline 4 & $B \cap \operatorname{Cn}(B \div \alpha) \subseteq B \div \alpha$ & relative closure \\
\hline 5 & If $\beta \in B$ then $[\beta \in B \div \alpha$ iff $\beta \in \operatorname{Cn}(B \div \alpha)]$ & 4 and Lemma 47 \\
\hline 6 & If $\beta \in B$ then $[\beta \notin B \div \alpha$ iff $\beta \notin \operatorname{Cn}(B \div \alpha)]$ & 5 (negating both sides of iff) \\
\hline 7 & $\alpha \leftrightharpoons \beta$ & Assumption \\
\hline 8 & $\beta \in B$ and $\beta \notin B \div \alpha$ & 2,7 \\
\hline 9 & $\beta \in B$ and $\beta \notin \operatorname{Cn}(B \div \alpha)$ & 6,8 \\
\hline 10 & {$[\beta \in B$ and $\beta \in \operatorname{Cn}(B)]$ and $\beta \notin \operatorname{Cn}(B \div \alpha)$} & $\begin{array}{l}1,9 \text { (adding redundant conjunct } \\
\beta \in \operatorname{Cn}(B) \text { ) }\end{array}$ \\
\hline 11 & $\beta \in B$ and $[\beta \in \operatorname{Cn}(B)$ and $\beta \notin \operatorname{Cn}(B \div \alpha)]$ & 10 \\
\hline 12 & $\beta \in B$ and $\alpha \leadsto \beta$ & 3,11 \\
\hline 13 & If $\alpha \leadsto \beta$ then $\beta \in B$ and $\alpha \leadsto \beta$ & 7,12 \\
\hline 14 & Since lines 7 through 12 are logically equivalen & , the reverse order also holds \\
\hline 15 & $\alpha \leadsto \beta$ iff $\beta \in B$ and $\alpha \leadsto \beta$ & 13,14 \\
\hline
\end{tabular}

Theorem 24. Given relations $\leadsto, \sim$ and $\div$ for belief base $B$ such that Cond $\leadsto$ and Cond $\leadsto$ hold respectively and closure is satisfied, in the case where $B$ is logically closed, $\leadsto$ reduces to $\rightarrow$ : $\alpha \leadsto \beta$ iff $\alpha \leadsto \beta$. 


\begin{tabular}{|c|c|c|}
\hline \multicolumn{3}{|c|}{ Proof. } \\
\hline 1 & $B \div \alpha \subseteq \operatorname{Cn}(B \div \alpha)$ & By inclusion for $\mathrm{Cn}$ \\
\hline 2 & $\alpha \leadsto \beta$ iff $\beta \in B$ and $\beta \notin B \div \alpha$ & Cond $\leadsto$ \\
\hline 3 & $\alpha \leadsto \beta$ iff $\beta \in \operatorname{Cn}(B)$ and $\beta \notin \operatorname{Cn}(B \div \alpha)$ & Cond $\leadsto$ \\
\hline 4 & If $\operatorname{Cn}(B) \subseteq B$ then $\operatorname{Cn}(B \div \alpha) \subseteq B \div \alpha$ & closure \\
\hline 5 & $B=\mathrm{Cn}(B)$ & Logical Closure \\
\hline 6 & $\operatorname{Cn}(B \div \alpha) \subseteq B \div \alpha$ & 4,5 \\
\hline 7 & $\beta \in B \div \alpha$ iff $\beta \in \operatorname{Cn}(B \div \alpha)$ & 1,6 \\
\hline 8 & $\alpha \leadsto \beta$ & Assumption \\
\hline 9 & $\beta \in B$ and $\beta \notin B \div \alpha$ & 2,8 \\
\hline 10 & $\beta \in \operatorname{Cn}(B)$ and $\beta \notin B \div \alpha$ & 5,9 \\
\hline 11 & $\beta \in \mathrm{Cn}(B)$ and $\beta \notin \operatorname{Cn}(B \div \alpha)$ & 7,10 \\
\hline 12 & $\alpha \leadsto \beta$ & 3,11 \\
\hline 13 & If $\alpha \leadsto \beta$ then $\alpha \leadsto \beta$ & 8,12 \\
\hline 14 & Since lines 8 through 12 are logically equivale & at, the reverse order also holds \\
\hline 15 & $\alpha \leadsto \beta$ iff $\alpha \leadsto \beta$ & 13,14 \\
\hline
\end{tabular}

\section{A.3 Proofs for $\S$ 3.5.3: Redundancy Resulting in Different Types of Base Dependence}

Theorem 27. Given relations $\sim$ and $\div$ for belief base $B$, where inclusion holds, Cond $\sim$ is equivalent to the following: $\quad \alpha \leadsto \beta$ iff $\beta$ is redundant in $B$ with respect to $B \div \alpha$.

\begin{tabular}{|c|c|c|}
\hline 1 & $B \div \alpha \subseteq B$ & inclusion \\
\hline 2 & $\alpha \stackrel{\sim}{\sim}$ iff $\beta$ is redundant in $B$ with respect to $B \div \alpha$ & Assume \\
\hline 3 & $\begin{array}{l}\alpha \check{\sim} \beta \text { iff } \\
\quad B \div \alpha \subseteq B \text { and } \beta \in B \text { and } \\
\quad \beta \notin B \div \alpha \text { and } \beta \in \operatorname{Cn}(B \div \alpha)\end{array}$ & $\begin{array}{l}2 \text { and Definition } 26 \\
\quad \text { (letting } B^{\prime}=B \div \alpha \text { ) }\end{array}$ \\
\hline 4 & $\begin{array}{l}\alpha \stackrel{\sim}{\sim} \beta \text { iff } \\
\quad \top \text { and } \beta \in B \text { and } \beta \notin B \div \alpha \text { and } \beta \in \operatorname{Cn}(B \div \alpha)\end{array}$ & $\begin{array}{l}1,3 \text { (replacing } B \div \alpha \subseteq B \\
\text { with } \top \text { as it is true by } 1 \text { ) }\end{array}$ \\
\hline 5 & $\alpha \check{\sim} \beta$ iff $\beta \in B$ and $\beta \notin B \div \alpha$ and $\beta \in \operatorname{Cn}(B \div \alpha)$ & $\begin{array}{l}4 \text { (removing conjunct } \top \text { ); } \\
\text { Cond } \sim \text { derived }\end{array}$ \\
\hline 6 & ince lines 2 through 5 are logically equivalent, the revers & e order also holds \\
\hline
\end{tabular}

Theorem 29. Given relations $\leadsto$ and $\div$ for belief base $B$ such that Cond $\sim$ holds, there is no weak base dependence if and only if relative closure holds for $\div$.

Proof.

Based on Definition 25, there is no weak base dependence if and only if $\alpha \dot{\psi} \beta \beta$ for all formulas $\alpha$ and $\beta$. In the following, we show that indeed relative closure holds if and only if $\alpha \ddot{\psi} \beta$ for all $\alpha$ and $\beta$. 


\begin{tabular}{l|l|l}
\hline 1 & $\alpha \check{\sim} \beta$ iff $\beta \in B$ and $\beta \notin B \div \alpha$ and $\beta \in \operatorname{Cn}(B \div \alpha)$ & Cond $\sim$ \\
2 & $\alpha \check{\sim} \beta$ iff $\beta \notin B$ or $\beta \in B \div \alpha$ or $\beta \notin \operatorname{Cn}(B \div \alpha)$ & 1 (negating both sides) \\
\hline 3 & $B \cap \operatorname{Cn}(B \div \alpha) \subseteq B \div \alpha$ & Assume relative closure \\
4 & If $\beta \in B$ and $\beta \in \operatorname{Cn}(B \div \alpha)$ then $\beta \in B \div \alpha$ & 3 (by the set theory) \\
5 & $\neg[\beta \in B$ and $\beta \in \operatorname{Cn}(B \div \alpha)]$ or $\beta \in B \div \alpha$ & 4 \\
6 & $\beta \notin B$ or $\beta \notin \operatorname{Cn}(B \div \alpha)$ or $\beta \in B \div \alpha$ & 5 \\
7 & $\alpha \check{\psi} \beta \beta$ & 2,6 ; so no weak base dep. exists \\
\hline 8 & Since lines 3 through 7 are logically equivalent, the reverse order also holds \\
\hline
\end{tabular}

\section{A.4 Proofs for $\S 4.1$ : Kernel Contraction and Base Dependence}

Lemma 30. In the presence of Def- $B$, Cond-ID ${ }^{B}$ and contribution, the following is equivalent to Cond $\stackrel{\overline{\div}}{\div} \quad \beta \in B \div \alpha$ iff $\beta \in B$ and $\alpha \not \nrightarrow \beta$.

\begin{tabular}{|c|c|c|}
\hline 1 & If $\beta \leadsto \beta$ then $\delta \leadsto \beta$ for some $\delta$ & Trivially holds: e.g., let $\delta$ be $\beta$ \\
\hline 2 & If $\delta \leadsto \beta$ then $\beta \leadsto \beta$ & Cond-ID ${ }^{B}$ \\
\hline 3 & $\beta \leadsto \beta$ iff $\delta \leadsto \beta$ for some $\delta$ & 1,2 \\
\hline 4 & $\beta \in B$ iff either $\beta \in \operatorname{Taut}(B)$ or $\delta \leftrightharpoons \beta$ for some $\delta$ & Def- $B$ \\
\hline 5 & If $\vdash \alpha \vee \beta$ then $\alpha \not \neg \beta$ & By Theorem 18 and contribution \\
\hline 6 & If $\vdash \beta$ then $\alpha \not \rightarrow \beta$ & 5 \\
\hline 7 & $\begin{array}{l}\beta \in B \div \alpha \text { iff } \\
\quad \text { either } \beta \in \operatorname{Taut}(B) \text { or } \beta \leadsto \beta \text { and } \alpha \not \Varangle \beta\end{array}$ & Assume Cond $\overline{\div}$ \\
\hline 8 & $\begin{array}{l}\beta \in B \div \alpha \text { iff } \\
\quad[\beta \in B \text { and } \vdash \beta] \text { or }[\beta \leadsto \beta \text { and } \alpha \not \neq \beta]\end{array}$ & 7 and Definition 15 \\
\hline 9 & $\begin{array}{l}\beta \in B \div \alpha \text { iff } \\
\quad[\beta \in B \text { and } \vdash \beta] \text { or } \\
\quad[\delta \lesssim \beta \text { for some } \delta] \text { and } \alpha \not \nrightarrow \beta]\end{array}$ & $\begin{array}{l}3,8 \text { (replacing } \beta \leftrightharpoons \beta \text { with its } \\
\quad \text { equivalent } \delta \leftrightharpoons \beta \text { for some } \delta \text { ) }\end{array}$ \\
\hline 10 & 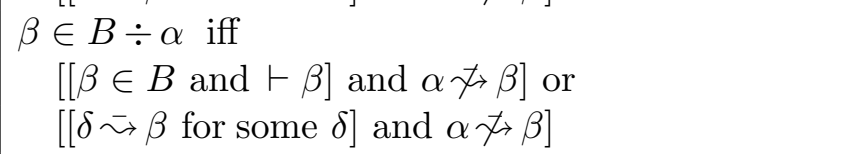 & $\begin{array}{l}6,9 \text { (adding redundant } \\
\quad \text { conjunct } \alpha \not \rightarrow \beta \text { since } \vdash \beta \text { ) }\end{array}$ \\
\hline 11 & $\begin{array}{l}\beta \in B \div \alpha \text { iff } \\
\quad[[\beta \in B \text { and } \vdash \beta] \text { or }[\delta \leadsto \beta \text { for some } \delta]] \\
\quad \text { and } \alpha \not{ }_{\star} \beta\end{array}$ & 10 \\
\hline 12 & $\begin{array}{l}\beta \in B \div \alpha \text { iff } \\
\quad[\beta \in \operatorname{Taut}(B) \text { or }[\delta \leadsto \beta \text { for some } \delta]] \text { and } \alpha \not \nrightarrow \beta\end{array}$ & 11 and Definition 15 \\
\hline 13 & $\beta \in B \div \alpha$ iff $\beta \in B$ and $\alpha \not \rightarrow \beta$ & 4,12 \\
\hline 14 & Since lines 7 through 13 are logically equivalent, the $r$ & erse order also holds \\
\hline
\end{tabular}

Lemma 31. Given relations $\leadsto$ and $\div$ for base $B$ such that Cond $\overline{\div}$ holds and contribution is satisfied, it also holds that: $\quad$ If $\beta \in B \div \alpha$ then $\alpha \not \nrightarrow \beta$. 


\begin{tabular}{|c|c|c|}
\hline \multicolumn{3}{|c|}{ Proof } \\
\hline 1 & If $\vdash \alpha \vee \beta$ then $\alpha \not \nrightarrow \beta$ & $\begin{array}{l}\text { By Theorem } 18 \\
\text { and contribution }\end{array}$ \\
\hline 2 & $\beta \in B \div \alpha$ iff either $\beta \in \operatorname{Taut}(B)$ or $\left(\beta \leadsto \beta\right.$ and $\left.\alpha \not{ }_{\star} \beta\right)$ & Cond $\div$ \\
\hline 3 & $\beta \in B \div \alpha$ & Assumption \\
\hline 4 & $\beta \in \operatorname{Taut}(B)$ or $[\beta \leftrightharpoons \beta$ and $\alpha \not \rightarrow \beta]$ & 2,3 \\
\hline 5 & $\beta \notin \operatorname{Taut}(B)$ & Case1 Assumption \\
\hline 6 & $\beta \stackrel{\sim}{\sim}$ and $\alpha \not \rightarrow \beta$ & 4,5 \\
\hline 7 & $\alpha \not \rightarrow \beta$ & 6 \\
\hline 8 & $\beta \in \operatorname{Taut}(B)$ & Case2 Assumption \\
\hline 9 & $\vdash \beta$ & 8 and Definition 15 \\
\hline 10 & $\alpha \not \rightarrow \beta$ & 1,9 \\
\hline 11 & $\alpha \not \rightarrow \beta$ & By Case1 and Case2 \\
\hline 12 & If $\beta \in B \div \alpha$ then $\alpha \not t \beta$ & 3,11 \\
\hline
\end{tabular}

Theorem 32 (Base Dependence to Base Contraction). Given relations $\leadsto$ and $\div$ for belief base $B$ such that Cond $\div$ holds, if $\leadsto$ is a base dependence, then $\div$ is a kernel contraction.

Proof.

Assume all the following hold: Cond $\overline{\div}$ and postulates of base dependence, namely, Def- $B$, Cond-ID ${ }^{B}$, conjugation, contribution and modularity (see Definition 19). We show that then the postulates of kernel contraction, viz., inclusion, success, uniformity and core-retainment (see §2.3.2) also hold:

$$
B \div \alpha \subseteq B
$$

(inclusion)

\begin{tabular}{|c|c|c|}
\hline 1 & $\beta \in B \div \alpha$ iff either $\beta \in \operatorname{Taut}(B)$ or $(\beta \leadsto \beta$ and $\alpha \not \neg \beta)$ & Cond $\stackrel{\overline{\div}}{\circ}$ \\
\hline 2 & $\beta \in B$ iff either $\beta \in \operatorname{Taut}(B)$ or $\alpha \leadsto \beta$ for some $\alpha$ & Def- $B$ \\
\hline 3 & $\beta \in B \div \alpha$ & Assumption \\
\hline 4 & $\beta \in \operatorname{Taut}(B)$ or $[\beta \leadsto \beta$ and $\alpha \not \Varangle \beta]$ & 1,3 \\
\hline 5 & $\beta \notin \operatorname{Taut}(B)$ & Case1 Assumption \\
\hline 6 & {$[\beta \leadsto \beta$ and $\alpha \not \beta \beta]$} & 4,5 \\
\hline 7 & $\beta \leadsto \beta$ & 6 (letting $\alpha$ be $\beta$ ) \\
\hline 8 & $\beta \in B$ & 2,7 \\
\hline 9 & $\beta \in \operatorname{Taut}(B)$ & Case2 Assumption \\
\hline 10 & $\beta \in B$ and $\vdash \beta$ & 9 and Definition 15 \\
\hline 11 & $\beta \in B$ & 10 \\
\hline 12 & $\beta \in B$ & By Case1 and Case2 \\
\hline 13 & If $\beta \in B \div \alpha$ then $\beta \in B$ & 3,12 \\
\hline 14 & $B \div \alpha \subseteq B$ & 13; inclusion derived \\
\hline
\end{tabular}




\begin{tabular}{|c|c|c|}
\hline 1 & If $\beta \in B \div \alpha$ then $\alpha \not \neg \beta$ & By Lemma 31 , contribution and Cond $\stackrel{\div}{-}$ \\
\hline 2 & $B \div \alpha \subseteq B$ & inclusion (proved above) \\
\hline 3 & $\begin{array}{l}\text { If } \alpha \in \operatorname{Cn}\left(B^{\prime}\right) \text { and } B^{\prime} \subseteq B \text { then } \\
\quad \text { either } \vdash \alpha \text { or } \alpha \leadsto \beta \text { for some } \beta \in B^{\prime}\end{array}$ & modularity \\
\hline 4 & $\alpha \in \operatorname{Cn}(B \div \alpha)$ & Assumption \\
\hline 5 & $\alpha \in \operatorname{Cn}(B \div \alpha)$ and $B \div \alpha \subseteq B$ & 2,4 \\
\hline 6 & $\vdash \alpha$ or $\alpha \leadsto \beta$ for some $\beta \in B \div \alpha$ & 3, 5 (letting $B^{\prime}=B \div \alpha$ ) \\
\hline 7 & $\vdash \alpha$ & 1,6 (as the second disjunct of 6 is false by 1 ) \\
\hline 8 & If $\alpha \in \operatorname{Cn}(B \div \alpha)$ then $\vdash \alpha$ & 4,7 \\
\hline 9 & If $\nvdash \alpha$ then $\alpha \notin \operatorname{Cn}(B \div \alpha)$ & 8; success derived \\
\hline
\end{tabular}

If $\alpha \in \operatorname{Cn}\left(B^{\prime}\right)$ iff $\beta \in \operatorname{Cn}\left(B^{\prime}\right)$ for all $B^{\prime} \subseteq B$ then $B \div \alpha=B \div \beta$

(uniformity)

\begin{tabular}{|c|c|c|}
\hline 1 & $\delta \in B \div \theta$ iff either $\delta \in \operatorname{Taut}(B)$ or $\delta \leadsto \delta$ and $\theta \not \nrightarrow \delta$ & Cond $\div$ \\
\hline 2 & $\begin{array}{l}\text { If } \alpha \in \operatorname{Cn}\left(B^{\prime}\right) \text { iff } \beta \in \operatorname{Cn}\left(B^{\prime}\right) \text { for all } B^{\prime} \subseteq B \\
\text { then } \alpha \leadsto \delta \text { iff } \beta \leadsto \delta\end{array}$ & conjugation \\
\hline 3 & $\alpha \in \operatorname{Cn}\left(B^{\prime}\right)$ iff $\beta \in \operatorname{Cn}\left(B^{\prime}\right)$ for all $B^{\prime} \subseteq B$ & Assumption \\
\hline 4 & $\alpha \leadsto \delta$ iff $\beta \leadsto \delta$ & 2,3 \\
\hline 5 & $\delta \in B \div \alpha$ & Assumption \\
\hline 6 & $\delta \in \operatorname{Taut}(B)$ or $[\delta \leadsto \delta$ and $\alpha \not \Varangle \delta]$ & 1,5 \\
\hline 7 & $\delta \notin \operatorname{Taut}(B)$ & Case1 Assumption \\
\hline 8 & {$[\delta \leadsto \delta$ and $\alpha \not \Varangle \delta]$} & 6,7 \\
\hline 9 & $\delta \leadsto \delta$ & 8 \\
\hline 10 & $\alpha \not 九 \delta$ & 8 \\
\hline 11 & $\beta \not \delta$ & 4,10 \\
\hline 12 & $\delta \leadsto \delta$ and $\beta \not \neg \delta$ & 9,11 \\
\hline 13 & $\delta \in B \div \beta$ & 1,12 \\
\hline 14 & $\delta \in \operatorname{Taut}(B)$ & Case2 Assumption \\
\hline 15 & $\delta \in B \div \beta$ & 1,14 \\
\hline 16 & $\delta \in B \div \beta$ & By Case1 and Case2 \\
\hline 17 & If $\delta \in B \div \alpha$ then $\delta \in B \div \beta$ & 5,16 \\
\hline 18 & $B \div \alpha \subseteq B \div \beta$ & 17 \\
\hline 19 & $B \div \beta \subseteq B \div \alpha$ & Also by symmetry (steps 5-18) \\
\hline 20 & $B \div \alpha=B \div \beta$ & 18,19 \\
\hline 21 & $\begin{array}{l}\text { If } \alpha \in \mathrm{Cn}\left(B^{\prime}\right) \text { iff } \beta \in \mathrm{Cn}\left(B^{\prime}\right) \text { for all } B^{\prime} \subseteq B \\
\text { then } B \div \alpha=B \div \beta\end{array}$ & 3,20 ; uniformity derived \\
\hline
\end{tabular}


If $\beta \in B$ and $\beta \notin B \div \alpha$ then there is some $B^{\prime}$ s.t.

$B^{\prime} \subseteq B$ and $\alpha \notin \operatorname{Cn}\left(B^{\prime}\right)$ and $\alpha \in \operatorname{Cn}\left(B^{\prime} \cup\{\beta\}\right)$

(core-retainment)

\begin{tabular}{|c|c|c|}
\hline 1 & $\beta \in B \div \alpha$ iff either $\beta \in \operatorname{Taut}(B)$ or $(\beta \leadsto \beta$ and $\alpha \not \nrightarrow \beta)$ & Cond $\stackrel{\overline{\div}}{\circ}$ \\
\hline 2 & $\beta \notin B \div \alpha$ iff $\beta \notin \operatorname{Taut}(B)$ and $[\beta \not \rightarrow \beta$ or $\alpha \leadsto \beta]$ & 1 (contrapositive) \\
\hline 3 & $\beta \in B$ iff either $\beta \in \operatorname{Taut}(B)$ or $\alpha \leadsto \beta$ for some $\alpha$ & Def- $B$ \\
\hline 4 & If $\alpha \leadsto \beta$ then $\beta \leadsto \beta$ & Cond-ID ${ }^{B}$ \\
\hline 5 & $\begin{array}{l}\text { If } \alpha \leadsto \beta \text { then } \\
\alpha \notin \operatorname{Cn}\left(B^{\prime}\right) \text { and } \alpha \in \operatorname{Cn}\left(B^{\prime} \cup\{\beta\}\right) \text { for some } B^{\prime} \subset B\end{array}$ & contribution \\
\hline 6 & $\beta \in B$ & Assumption \\
\hline 7 & $\beta \notin B \div \alpha$ & Assumption \\
\hline 8 & $\beta \in \operatorname{Taut}(B)$ or $\alpha \leadsto \beta$ for some $\alpha$ & 3,6 \\
\hline 9 & $\beta \notin \operatorname{Taut}(B)$ and $[\beta \not \rightarrow \beta$ or $\alpha \stackrel{\sim}{\sim}]$ & 2,7 \\
\hline 10 & $\beta \notin \operatorname{Taut}(B)$ & 9 \\
\hline 11 & $\alpha \stackrel{\sim}{\sim}$ for some $\alpha$ & 8,10 \\
\hline 12 & $\beta \approx \beta$ & 4,11 \\
\hline 13 & {$[\beta \not \neg \beta$ or $\alpha \leadsto \beta]$} & 9 \\
\hline 14 & $\alpha \stackrel{\sim}{\sim}$ & 12,13 \\
\hline 15 & $\alpha \notin \operatorname{Cn}\left(B^{\prime}\right)$ and $\alpha \in \operatorname{Cn}\left(B^{\prime} \cup\{\beta\}\right)$ for some $B^{\prime} \subseteq B$ & 5,14 \\
\hline 16 & $\begin{array}{l}\text { If } \beta \in B \text { and } \beta \notin B \div \alpha \text { then } \\
\alpha \notin \operatorname{Cn}\left(B^{\prime}\right) \text { and } \alpha \in \operatorname{Cn}\left(B^{\prime} \cup\{\beta\}\right) \text { for some } B^{\prime} \subseteq B\end{array}$ & $\begin{array}{l}6,7,15 \\
\quad \text { core-retainment derived }\end{array}$ \\
\hline
\end{tabular}

Theorem 33 (Base Contraction to Base Dependence). Given relations $\leadsto$ and $\div$ for belief base $B$ such that Cond $\leadsto$ holds, if $\div$ is a kernel contraction, then $\leadsto$ is a base dependence.

Proof.

Assume all the following hold: Cond $\leadsto$ and the postulates for kernel contraction, namely, inclusion, success, uniformity and core-retainment (see $\S 2.3 .2$ ). We show that then the postulates of base dependence, viz., Def- $B$, Cond-ID ${ }^{B}$, conjugation, contribution and modularity (see Definition 19) also hold:

If $\alpha \in \mathrm{Cn}\left(B^{\prime}\right)$ iff $\beta \in \mathrm{Cn}\left(B^{\prime}\right)$ for all $B^{\prime} \subseteq B$ then $\alpha \leftrightharpoons \delta$ iff $\beta \leftrightharpoons \delta$

(conjugation)

\begin{tabular}{|c|c|c|}
\hline 1 & $\theta \leadsto \delta$ iff $\delta \in B$ and $\delta \notin B \div \theta$ & Cond $\leadsto$ \\
\hline 2 & If $\alpha \in \operatorname{Cn}\left(B^{\prime}\right)$ iff $\beta \in \operatorname{Cn}\left(B^{\prime}\right)$ for all $B^{\prime} \subseteq B$ then $B \div \alpha=B \div \beta$ & uniformity \\
\hline 3 & $\alpha \in \operatorname{Cn}\left(B^{\prime}\right)$ iff $\beta \in \operatorname{Cn}\left(B^{\prime}\right)$ for all $B^{\prime} \subseteq \bar{B}$ & Assumption \\
\hline 4 & $B \div \alpha=B \div \beta$ & 2,3 \\
\hline 5 & $\delta \in B \div \alpha$ iff $\delta \in B \div \beta$ & 4 \\
\hline 6 & $\delta \notin B \div \alpha$ iff $\delta \notin B \div \beta$ & 5 \\
\hline 7 & {$[\delta \in B$ and $\delta \notin B \div \alpha]$ iff $[\delta \in B$ and $\delta \notin B \div \beta]$} & $\begin{array}{l}6 \text { (adding conjunct } \\
\delta \in B \text { to both sides) }\end{array}$ \\
\hline 8 & $\alpha \leadsto \delta$ iff $\beta \leftrightharpoons \delta$ & 1,7 \\
\hline 9 & If $\alpha \in \operatorname{Cn}\left(B^{\prime}\right)$ iff $\beta \in \operatorname{Cn}\left(B^{\prime}\right)$ for all $B^{\prime} \subseteq B$ then $\alpha \stackrel{\sim}{\sim}$ iff $\beta \leadsto \delta$ & $\begin{array}{l}3,8 ; \\
\quad \text { conjugation derived }\end{array}$ \\
\hline
\end{tabular}


If $\alpha \leadsto \beta$ then $\beta \leadsto \beta$

(Cond-ID ${ }^{B}$ )

\begin{tabular}{|c|c|c|}
\hline 1 & $\alpha \leadsto \beta$ iff $\beta \in B$ and $\beta \notin B \div \alpha$ & Cond $\leadsto$ \\
\hline 2 & $\alpha \not 九 \beta$ iff $\beta \notin B$ or $\beta \in B \div \alpha$ & 1 (negating both sides) \\
\hline 3 & If $\beta \in \operatorname{Cn}(B \div \beta)$ then $\beta \in \operatorname{Cn}(\emptyset)$ & success (contrapositive) \\
\hline 4 & $B \cap \operatorname{Cn}(B \div \alpha) \subseteq B \div \alpha$ & relative closure \\
\hline 5 & $\beta \not \rightarrow \beta$ & Assumption \\
\hline 6 & $\beta \notin B$ or $\beta \in B \div \beta$ & 2,5 \\
\hline 7 & $\beta \in B$ & Case1 Assumption \\
\hline 8 & $\beta \in B \div \beta$ & 6,7 \\
\hline 9 & $\beta \in \operatorname{Cn}(B \div \beta)$ & 8 , inclusion for $\mathrm{Cn}$ \\
\hline 10 & $\beta \in \operatorname{Cn}(\emptyset)$ & 3,9 \\
\hline 11 & $\beta \in \operatorname{Cn}(B \div \alpha)$ & 10 , monotony for $\mathrm{Cn}$ \\
\hline 12 & $\beta \in B \div \alpha$ & $4,7,11$ \\
\hline 13 & $\alpha \not \rightarrow \beta$ & 2,12 \\
\hline 14 & $\beta \notin B$ & Case 2 Assumption \\
\hline 15 & $\alpha \not \rightarrow \beta$ & 2,14 \\
\hline 16 & $\alpha \not \rightarrow \beta$ & By Case1 and Case2 \\
\hline 17 & If $\beta \not \rightarrow \beta$ then $\alpha \not \rightarrow \beta$ & 5,16 \\
\hline 18 & If $\alpha \leadsto \beta$ then $\beta \leadsto \beta$ & 17; Cond-ID ${ }^{B}$ derived \\
\hline
\end{tabular}

If $\alpha \in \operatorname{Cn}\left(B^{\prime}\right)$ and $B^{\prime} \subseteq B$ then either $\vdash \alpha$ or $\alpha \leadsto \beta$ for some $\beta \in B^{\prime}$

(modularity)

\begin{tabular}{|c|c|c|}
\hline 1 & $\alpha \leadsto \beta$ iff $\beta \in B$ and $\beta \notin B \div \alpha$ & Cond $\leadsto$ \\
\hline 2 & $\alpha \not \neg \beta$ iff $\beta \notin B$ or $\beta \in B \div \alpha$ & 1 \\
\hline 3 & If $\nvdash \alpha$ then $\alpha \notin \operatorname{Cn}(B \div \alpha)$ & success \\
\hline 4 & If $\alpha \in \operatorname{Cn}(B \div \alpha)$ then $\vdash \alpha$ & 3 \\
\hline 5 & $\alpha \in \operatorname{Cn}\left(B^{\prime}\right)$ & Assumption \\
\hline 6 & $B^{\prime} \subseteq B$ & Assumption \\
\hline 7 & $\beta \in B$ for all $\beta \in B^{\prime}$ & 6 (by the set theory) \\
\hline 8 & $\neg\left[\vdash \alpha\right.$ or $\alpha \leadsto \beta$ for some $\left.\beta \in B^{\prime}\right]$ & Assume for the sake of contradiction \\
\hline 9 & $\forall \alpha$ and $\alpha \not \rightarrow \beta$ for all $\beta \in B^{\prime}$ & 8 \\
\hline 10 & $\forall \alpha$ & 9 \\
\hline 11 & $\alpha \not \neg \beta$ for all $\beta \in B^{\prime}$ & 9 \\
\hline 12 & {$[\beta \notin B$ or $\beta \in B \div \alpha]$ for all $\beta \in B^{\prime}$} & 2,11 \\
\hline 13 & {$[\beta \in B \div \alpha]$ for all $\beta \in B^{\prime}$} & 7,12 (i.e., $\beta \notin B$ is false by 7 ) \\
\hline 14 & $B^{\prime} \subseteq B \div \alpha$ & 13 (by the set theory) \\
\hline 15 & $\operatorname{Cn}\left(B^{\prime}\right) \subseteq \operatorname{Cn}(B \div \alpha)$ & 14 , monotony for $\mathrm{Cn}$ \\
\hline 16 & $\alpha \in \operatorname{Cn}(B \div \alpha)$ & 5,15 \\
\hline 17 & $\vdash \alpha$ & 3,14 \\
\hline 18 & $\forall \alpha$ and $\vdash \alpha$ & 10,17 \\
\hline 19 & $\perp \quad$ (i.e., reached a contradiction) & 18 \\
\hline 20 & $\vdash \alpha$ or $\alpha \leadsto \beta$ for some $\beta \in B^{\prime}$ & 8,19 \\
\hline 21 & $\begin{array}{l}\text { If } \alpha \in \operatorname{Cn}\left(B^{\prime}\right) \text { and } B^{\prime} \subseteq B \text { then } \\
\text { either } \vdash \alpha \text { or } \alpha \stackrel{\sim}{\sim} \beta \text { for some } \beta \in B^{\prime}\end{array}$ & $5,6,20 ;$ modularity derived \\
\hline
\end{tabular}




\begin{tabular}{|c|c|c|}
\hline 1 & $B \div \alpha \subseteq \operatorname{Cn}(B \div \alpha)$ & By inclusion for $\mathrm{Cn}$ \\
\hline 2 & If $\beta \notin \operatorname{Cn}(B \div \alpha)$ then $\beta \notin B \div \alpha$ & 1 \\
\hline 3 & $\alpha \leadsto \beta$ iff $\beta \in B$ and $\beta \notin B \div \alpha$ & Cond $\leadsto$ \\
\hline 4 & If $\nvdash \beta$ then $\beta \notin \operatorname{Cn}(B \div \beta)$ & success \\
\hline 5 & $\beta \in B$ & Assumption, for left to right \\
\hline 6 & $\vdash \beta \vee \nvdash \beta$ & Tautological truth \\
\hline 7 & $\vdash \beta$ & Case1 Assumption \\
\hline 8 & $\beta \in B$ and $\vdash \beta$ & 5,7 \\
\hline 9 & $\beta \in \operatorname{Taut}(B)$ & 8 and Definition 15 \\
\hline 10 & $\nvdash \beta$ & Case2 Assumption \\
\hline 11 & $\beta \notin \mathrm{Cn}(B \div \beta)$ & 4,10 \\
\hline 12 & $\beta \notin B \div \beta$ & 2,11 \\
\hline 13 & $\beta \in B$ and $\beta \notin B \div \beta$ & 5,12 \\
\hline 14 & $\beta \leadsto \beta$ & 3,13 \\
\hline 15 & $\alpha \leadsto \beta$ for some $\alpha$ & 14 (e.g., let $\alpha$ be equal to $\beta$ ) \\
\hline 16 & $\beta \in \operatorname{Taut}(B)$ or $\alpha \leadsto \beta$ for some $\alpha$ & By 6, Case1 and Case2 \\
\hline 17 & If $\beta \in B$ then $[\beta \in \operatorname{Taut}(B)$ or $\alpha \leadsto \beta$ for some $\alpha]$ & 5,16 \\
\hline 18 & $\beta \notin B$ & Assumption, for right to left \\
\hline 19 & $\beta \notin \operatorname{Taut}(B)$ & 18 and Definition 15 \\
\hline 20 & $\alpha \not \rightarrow \beta$ for all $\alpha$ & 3,18 \\
\hline 21 & $\beta \notin \operatorname{Taut}(B)$ and $\alpha \not \rightarrow \beta$ for all $\alpha$ & 19,20 \\
\hline 22 & If $\beta \notin B$ then $[\beta \notin \operatorname{Taut}(B)$ and $\alpha \not \rightarrow \beta$ for all $\alpha]$ & 18,21 \\
\hline 23 & If $[\beta \in \operatorname{Taut}(B)$ or $\alpha \leadsto \beta$ for some $\alpha]$ then $\beta \in B$ & 22 (contrapositive) \\
\hline 24 & $\beta \in B$ iff either $\beta \in \operatorname{Taut}(B)$ or $\alpha \leadsto \beta$ for some $\alpha$ & 17, 23; Def- $B$ derived \\
\hline
\end{tabular}

If $\alpha \leadsto \beta$ then $\alpha \notin \operatorname{Cn}\left(B^{\prime}\right)$ and $\alpha \in \operatorname{Cn}\left(B^{\prime} \cup\{\beta\}\right)$ for some $B^{\prime} \subseteq B$

(contribution)

\begin{tabular}{c|l|l}
\hline 1 & $\alpha \leadsto \beta$ iff $\beta \in B$ and $\beta \notin B \div \alpha$ & Cond $\leadsto$ \\
2 & If $\beta \in B$ and $\beta \notin B \div \alpha$ then & core-retainment \\
& $\alpha \notin \operatorname{Cn}\left(B^{\prime}\right)$ and $\alpha \in \operatorname{Cn}\left(B^{\prime} \cup\{\beta\}\right)$ for some $B^{\prime} \subseteq B$ & \\
\hline 3 & $\alpha \leadsto \beta$ & Assumption \\
4 & $\beta \in B$ and $\beta \notin B \div \alpha$ & 1,3 \\
5 & $\alpha \notin \operatorname{Cn}\left(B^{\prime}\right)$ and $\alpha \in \operatorname{Cn}\left(B^{\prime} \cup\{\beta\}\right)$ for some $B^{\prime} \subseteq B$ & 2,4 \\
6 & If $\alpha \cong \beta$ then & 3,5 ; contribution derived \\
& $\alpha \notin \operatorname{Cn}\left(B^{\prime}\right)$ and $\alpha \in \operatorname{Cn}\left(B^{\prime} \cup\{\beta\}\right)$ for some $B^{\prime} \subseteq B$ & \\
\hline
\end{tabular}

Theorem 34 (Characterization). Let the relations $\leadsto$ and $\div$ for belief base B be such that $\div$ satisfies inclusion, and that Cond $\leadsto$ holds. Then, $\div$ is a kernel contraction if and only if $\leadsto$ is a base dependence. 
Proof.

Given that Cond $\leadsto$ holds by assumption, the left to right direction is already proved in Theorem 33 (§A.4). Similarly, for the right to left direction, Theorem 32 (§A.4) can be used, provided that Cond $\overline{\div}$ holds. That is, to construct a kernel contraction relation $\div$, given a base dependence relation $\leadsto$, it suffices to show that Cond $\bar{\doteqdot}$ holds. To achieve this, let us assume all the following hold:

- three of the base dependence axioms (see Definition 19), namely, Def- $B$, Cond-ID ${ }^{B}$ and contribution,

- Cond $\leadsto$, and

- inclusion (see Remark 10).

\begin{tabular}{|c|c|c|}
\hline 1 & $B \div \alpha \subseteq B$ & inclusion \\
\hline 2 & $\alpha \leadsto \beta$ iff $\beta \in B$ and $\beta \notin B \div \alpha$ & Cond $\leadsto$ \\
\hline 3 & $\beta \in B \div \alpha$ & Assumption \\
\hline 4 & $\beta \in B$ & 1,3 \\
\hline 5 & $\alpha \not t \beta$ & 2,3 \\
\hline 6 & If $\beta \in B \div \alpha$ then $\beta \in B$ and $\alpha \not \rightarrow \beta$ & $3,4,5$ \\
\hline 7 & If $\beta \in B$ and $\beta \notin B \div \alpha$ then $\alpha \leadsto \beta$ & 2 (using right to left) \\
\hline 8 & If $\beta \notin B \div \alpha$ then $\beta \notin B$ or $\alpha \stackrel{\sim}{\sim}$ & 7 \\
\hline 9 & If $\beta \in B$ and $\alpha \not \rightarrow \beta$ then $\beta \in B \div \alpha$ & 8 (contrapositive) \\
\hline 10 & $\beta \in B \div \alpha$ iff $\beta \in B$ and $\alpha \not \rightarrow \beta$ & 6,9 \\
\hline 11 & $\beta \in B \div \alpha$ iff either $\beta \in \operatorname{Taut}(B)$ or $\beta \leftrightharpoons \beta$ and $\alpha \not \rightarrow \beta$ & $\begin{array}{l}10 \text { and Lemma } 30 ; \\
\text { Cond } \overline{\div} \text { derived }\end{array}$ \\
\hline
\end{tabular}

Lemma 30 is applicable on line 11 because Def- $B$, Cond-ID ${ }^{B}$ and contribution are assumed.

\section{A.5 Proofs for $\S$ 4.2: Saturated Kernel Contraction and Strong Base Dependence}

Theorem 35 (Base Dependence to Base Contraction). Given relations $\approx$ and $\div$ for belief base $B$ such that Cond $\div$ holds, if $\leadsto$ is a strong base dependence, then $\div$ is a saturated kernel contraction.

Proof.

Assuming that Cond $\div$ holds and that $\leadsto$ is a base dependence, then $\div$ is a kernel contraction by Theorem 32 (§A.4). Further assume that $\leadsto$ also satisfies redundancy, which means $\leftrightharpoons$ is a strong base dependence relation (see Definition 20). We show that then $\div$ also satisfies relative closure, meaning $\div$ is a saturated kernel contraction (see $\S 2.3 .2$ ).

$$
B \cap \operatorname{Cn}(B \div \alpha) \subseteq B \div \alpha
$$

(relative closure)

\begin{tabular}{|c|c|c|}
\hline 1 & If $\beta \in B \div \alpha$ then $\alpha \not \Varangle \beta$ & $\begin{array}{l}\text { By Lemma } 31 \text {, contribution } \\
\text { and Cond } \stackrel{\dot{*}}{ }\end{array}$ \\
\hline 2 & $B \div \alpha \subseteq B$ & inclusion by Theorem 32 \\
\hline 3 & $\beta \in B \div \alpha$ iff either $\beta \in \operatorname{Taut}(B)$ or $\beta \leadsto \beta$ and $\alpha \not \Varangle \beta$ & Cond $\overline{\div}$ \\
\hline 4 & $\beta \in B$ iff either $\beta \in \operatorname{Taut}(B)$ or $\alpha \leadsto \beta$ for some $\alpha$ & Def- $B$ \\
\hline 5 & If $\alpha \leadsto \beta$ then $\beta \leadsto \beta$ & Cond-ID ${ }^{B}$ \\
\hline 6 & $\begin{array}{l}\text { If } \beta \in \operatorname{Cn}\left(B^{\prime}\right) \text { and } B^{\prime} \subseteq B \text { then } \\
\quad \text { either } \alpha \not \beta \text { or } \alpha \leadsto \delta \text { for some } \delta \in B^{\prime}\end{array}$ & redundancy \\
\hline
\end{tabular}




\begin{tabular}{|c|c|c|}
\hline 7 & If $\vdash \alpha \vee \beta$ then $\alpha \not \nrightarrow \beta$ & By Thm. 18 and contribution \\
\hline 8 & If $\vdash \beta$ then $\alpha \not \rightarrow \beta$ & 7 \\
\hline 9 & If $\vdash \beta$ and $\beta \in B$ then $\alpha \not \star \beta$ & $\begin{array}{l}8 \text { (introducing extra conjunct } \\
\beta \in B \text { to antecedent) }\end{array}$ \\
\hline 10 & If $\beta \in \operatorname{Taut}(B)$ then $\alpha \not \nrightarrow \beta$ & 9 and Definition 15 \\
\hline 11 & $\beta \in B$ & Assumption \\
\hline 12 & $\beta \in \operatorname{Taut}(B)$ or $\alpha \leadsto \beta$ for some $\alpha$ & 4,11 \\
\hline 13 & $\beta \in \operatorname{Taut}(B)$ or $\beta \leadsto \beta$ & 5,12 \\
\hline 14 & $\beta \in \operatorname{Cn}(B \div \alpha)$ & Assumption \\
\hline 15 & $\beta \in \mathrm{Cn}(B \div \alpha)$ and $B \div \alpha \subseteq B$ & 2,14 \\
\hline 16 & $\alpha \not \Varangle \beta$ or $\alpha \leadsto \delta$ for some $\delta \in B \div \alpha$ & 6,15 (letting $B^{\prime}=B \div \alpha$ ) \\
\hline 17 & $\alpha \not \rightarrow \beta$ & $\begin{array}{l}1,16 \text { (as the second disjunct } \\
\text { of } 16 \text { is false by } 1 \text { ) }\end{array}$ \\
\hline 18 & {$[\beta \in \operatorname{Taut}(B)$ or $\beta \leadsto \beta]$ and $[\alpha \not \rightarrow \beta]$} & 13,17 \\
\hline 19 & {$[\beta \in \operatorname{Taut}(B)$ and $\alpha \not \rightarrow \beta]$ or $[\beta \leadsto \beta$ and $\alpha \not \rightarrow \beta]$} & 18 (distributing conjunction) \\
\hline 20 & {$[\beta \in \operatorname{Taut}(B)]$ or $[\beta \leadsto \beta$ and $\alpha \not \nrightarrow \beta]$} & $\begin{array}{l}\text { 10, } 19 \text { (omitting redundant } \\
\text { conjunct } \alpha \not \Varangle \beta \text { ) }\end{array}$ \\
\hline 21 & $\beta \in B \div \alpha$ & 3,20 \\
\hline 22 & If $\beta \in B$ and $\beta \in \operatorname{Cn}(B \div \alpha)$ then $\beta \in B \div \alpha$ & $11,14,21$ \\
\hline 23 & $B \cap \mathrm{Cn}(B \div \alpha) \subseteq B \div \alpha$ & 22 ; relative closure derived \\
\hline
\end{tabular}

Theorem 36 (Base Contraction to Base Dependence). Given relations $\leadsto$ and $\div$ for belief base $B$ such that Cond $\leadsto$ holds, if $\div$ is a saturated kernel contraction, then $\leadsto$ is a strong base dependence.

Proof.

Assuming that Cond $\leadsto$ holds and that $\div$ is a kernel contraction, then $\leadsto$ is a base dependence by Theorem 33 (§A.4). Further assume that $\div$ also satisfies relative closure, which means $\div$ is a saturated kernel contraction (see $\S 2.3 .2$ ). We show that then that $\leadsto$ also satisfies redundancy, which means $\leadsto$ is a strong base dependence relation (see Definition 20).

If $\beta \in \operatorname{Cn}\left(B^{\prime}\right)$ and $B^{\prime} \subseteq B$ then either $\alpha \not \Varangle \beta$ or $\alpha \leftrightharpoons \delta$ for some $\delta \in B^{\prime} \quad$ (redundancy)

\begin{tabular}{r|l|l}
\hline 1 & $\alpha \leadsto \beta$ iff $\beta \in B$ and $\beta \notin B \div \alpha$ & Cond $~$ \\
2 & $\alpha \nsubseteq \beta$ iff $\beta \notin B$ or $\beta \in B \div \alpha$ & 1 \\
3 & $B \cap \operatorname{Cn}(B \div \alpha) \subseteq B \div \alpha$ & relative closure \\
\hline 4 & $\beta \in \operatorname{Cn}\left(B^{\prime}\right)$ & Assumption \\
5 & $B^{\prime} \subseteq B$ & Assumption \\
6 & $\delta \in B$ for all $\delta \in B^{\prime}$ & 5 (by the set theory) \\
7 & $\neg\left[\alpha \sim \delta\right.$ for some $\left.\delta \in B^{\prime}\right]$ & Assumption \\
8 & $\alpha \not \Varangle \delta$ for all $\delta \in B^{\prime}$ & 7 \\
9 & {$[\delta \notin B$ or $\delta \in B \div \alpha]$ for all $\delta \in B^{\prime}$} & 2,8 \\
10 & {$[\delta \in B \div \alpha]$ for all $\delta \in B^{\prime}$} & 6,9 \\
11 & $B^{\prime} \subseteq B \div \alpha$ & 10 (by the set theory) \\
12 & $\operatorname{Cn}\left(B^{\prime}\right) \subseteq \operatorname{Cn}(B \div \alpha)$ & 11, monotony for Cn
\end{tabular}




\begin{tabular}{|c|c|c|}
\hline 13 & $\beta \in \operatorname{Cn}(B \div \alpha)$ & 4,12 \\
\hline 14 & $\beta \in B$ & Case1 Assumption \\
\hline 15 & $\beta \in B \div \alpha$ & $3,13,14$ \\
\hline 6 & $\alpha \not \rightarrow \beta$ & 2,15 \\
\hline 17 & $\beta \notin B$ & Case2 Assumption \\
\hline 18 & $\alpha \not \rightarrow \beta$ & 2,17 \\
\hline 19 & $\alpha \not \rightarrow \beta$ & By Case1 and Case2 \\
\hline 20 & $\begin{array}{l}\text { If } \beta \in \operatorname{Cn}\left(B^{\prime}\right) \text { and } B^{\prime} \subseteq B \text { and } \\
\quad \neg\left[\alpha \approx \delta \text { for some } \delta \in B^{\prime}\right] \text { then } \alpha \not \beta \beta\end{array}$ & $4,5,7,19$ \\
\hline 21 & $\begin{array}{l}\text { If } \beta \in \operatorname{Cn}\left(B^{\prime}\right) \text { and } B^{\prime} \subseteq B \text { then } \\
\alpha \neq \beta \text { or } \alpha \cong \delta \text { for some } \delta \in B^{\prime}\end{array}$ & 20; redundancy derived \\
\hline
\end{tabular}

Theorem 37 (Characterization). Let the relations $\leadsto$ and $\div$ for belief base B be such that $\div$ satisfies inclusion and that Cond $\leadsto$ holds. Then, $\div$ is a saturated kernel contraction if and only if $\leadsto$ is a strong base dependence.

Proof.

This axiomatic characterization theorem proof closely resembles the proof of the characterization Theorem 34 (§A.4). The only difference is that here for the right to left direction, we need to use Theorem 35, and for the left to right direction, we use Theorem 36. Everything else stays the same, and thus is omitted here for brevity.

\section{A.6 Proofs for $\S$ 4.3: Factoring in Composite Dependence}

Theorem 39. If a relation $\leadsto$ satisfies $\mathrm{CCDF}^{B}$, then it also satisfies both $\mathrm{CCD}^{B}$ and $\mathrm{CCI}^{B}$.

Proof.

\begin{tabular}{|c|c|c|}
\hline 1 & 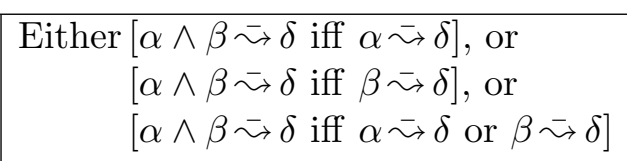 & $\mathrm{CCDF}^{B}$ \\
\hline 2 & $\alpha \leadsto \delta$ and $\beta \approx \delta$ & Assumption \\
\hline 3 & $\alpha \leadsto \delta$ & 2 \\
\hline 4 & $\beta \leadsto \delta$ & 2 \\
\hline 5 & $\alpha \leftrightharpoons \delta$ or $\beta \leftrightharpoons \delta$ & 3,4 \\
\hline 6 & $\alpha \wedge \beta \leftrightharpoons \delta$ & $1,3,4,5$ \\
\hline 7 & If $\alpha \leftrightharpoons \delta$ and $\beta \leadsto \delta$ then $\alpha \wedge \beta \leadsto \delta$ & 2,$6 ; \mathrm{CCD}^{B}$ derived \\
\hline
\end{tabular}


From $\mathrm{CCDF}^{B}$ to $\mathrm{CCl}^{B}$ :

\begin{tabular}{|c|c|c|}
\hline 1 & $\begin{array}{l}\text { Either }[\alpha \wedge \beta \leadsto \delta \text { iff } \alpha \leadsto \delta], \text { or } \\
{[\alpha \wedge \beta \approx \delta \text { iff } \beta \leftrightharpoons \delta], \text { or }} \\
{[\alpha \wedge \beta \leadsto \delta \text { iff } \alpha \leadsto \delta \text { or } \beta \leftrightharpoons \delta]}\end{array}$ & $\mathrm{CCDF}^{B}$ \\
\hline 2 & $\alpha \wedge \beta \leadsto \delta$ & Assumption \\
\hline 3 & {$[\alpha \leftrightharpoons \delta]$ or $[\beta \leftrightharpoons \delta]$ or $[\alpha \leftrightharpoons \delta$ or $\beta \leftrightharpoons \delta]$} & 1,2 \\
\hline 4 & $\alpha \leadsto \delta$ or $\beta \leadsto \delta$ & 3 \\
\hline 5 & If $\alpha \leadsto \delta$ or $\beta \leadsto \delta$ then $\alpha \wedge \beta \leadsto \delta$ & 2,4 \\
\hline 6 & If $\alpha \not \rightarrow \delta$ and $\beta \not \rightarrow \delta$ then $\alpha \wedge \beta \not \rightarrow \delta$ & 5 (contrapositive); $\mathrm{CCl}^{B}$ derived \\
\hline
\end{tabular}

Theorem 40 (Base Dependence to Base Contraction). Given relations $\leadsto$ and $\div$ for belief base $B$ such that Cond $\doteqdot$ holds, if $\leadsto$ is a strong base dependence that satisfies $\mathrm{CCDF}^{B}$, then $\div$ is a saturated kernel contraction that satisfies conjunctive factoring.

Proof.

Assuming that Cond $\overline{\div}$ holds and that $\approx$ is a strong base dependence, then $\div$ is a saturated kernel contraction by Theorem 35 (§A.5). Further assume that $\leadsto$ also satisfies CCDF $^{B}$. In the following, we show that then $\div$ also satisfies conjunctive factoring.

\begin{tabular}{|c|c|c|}
\hline 1 & $\delta \in B \div \theta$ iff either $\delta \in \operatorname{Taut}(B)$ or $\delta \leadsto \delta$ and $\theta \not \nrightarrow \delta$ & Cond $\div$ \\
\hline 2 & 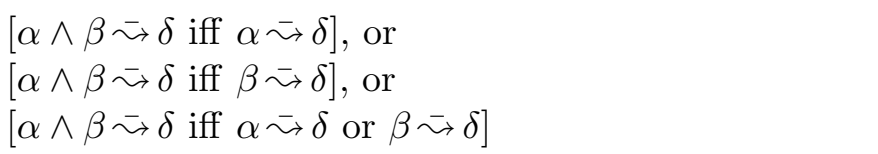 & $\begin{array}{l}\text { Assume } \mathrm{CCDF}^{B} \\
\text { (the word "Either" is omitted } \\
\text { to save space) }\end{array}$ \\
\hline 3 & $\begin{array}{l}{[\alpha \wedge \beta \not \rightarrow \delta \text { iff } \alpha \not \rightarrow \delta], \text { or }} \\
{[\alpha \wedge \beta \not \rightarrow \delta \text { iff } \beta \not \nrightarrow \delta], \text { or }} \\
{[\alpha \wedge \beta \not \rightarrow \delta \text { iff } \alpha \not \nrightarrow \delta \text { and } \beta \not \rightarrow \delta]}\end{array}$ & $\begin{array}{l}2 \text { (negating both sides of each } \\
\text { iff statement) }\end{array}$ \\
\hline 4 & 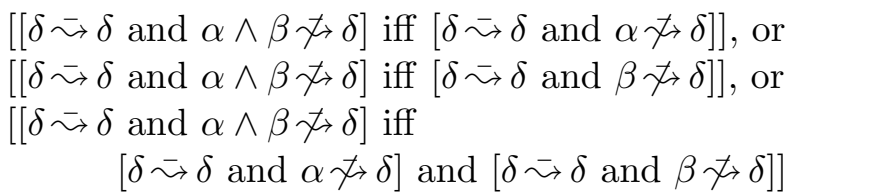 & $\begin{array}{l}3 \text { (adding the conjunct } \\
\delta \lesssim \delta \text { to both sides } \\
\text { of each iff statement) }\end{array}$ \\
\hline 5 & 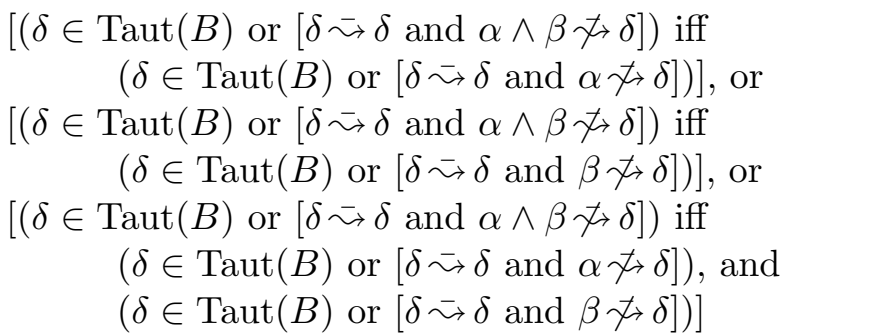 & $\begin{array}{l}4 \text { (adding the disjunct } \\
\delta \in \operatorname{Taut}(B) \text { to both sides } \\
\text { of each iff statement) }\end{array}$ \\
\hline 6 & $\begin{array}{l}{[\delta \in B \div \alpha \wedge \beta \text { iff } \delta \in B \div \alpha], \text { or }} \\
{[\delta \in B \div \alpha \wedge \beta \text { iff } \delta \in B \div \beta], \text { or }} \\
{[\delta \in B \div \alpha \wedge \beta \text { iff } \delta \in B \div \alpha \text { and } \delta \in B \div \beta]}\end{array}$ & 1,5 \\
\hline 7 & $\begin{array}{l}B \div \alpha \wedge \beta=B \div \alpha, \text { or } \\
B \div \alpha \wedge \beta=B \div \beta, \text { or } \\
B \div \alpha \wedge \beta=B \div \alpha \cap B \div \beta\end{array}$ & $\begin{array}{l}6 \text { (by the set theory); } \\
\text { conjunctive factoring derived }\end{array}$ \\
\hline
\end{tabular}


Theorem 41 (Base Contraction to Base Dependence). Given relations $\leadsto$ and $\div$ for belief base $B$ such that Cond $\leadsto$ holds, if $\div$ is a saturated kernel contraction that satisfies conjunctive factoring, then $\leadsto$ is a strong base dependence that satisfies $\mathrm{CCDF}^{B}$.

Proof.

Assuming that Cond $\leftrightharpoons$ holds and that $\div$ is a saturated kernel contraction, then $\leadsto$ is a strong base dependence by Theorem 36 (§A.5). Further, assume that $\div$ also satisfies conjunctive factoring. In the following, we show that then $\leadsto$ also satisfies $\mathrm{CCDF}^{B}$.

\begin{tabular}{|c|c|c|}
\hline 1 & $\theta \leadsto \delta$ iff $\delta \in B$ and $\delta \notin B \div \theta$ & Cond $\leadsto$ \\
\hline 2 & $\begin{array}{l}B \div \alpha \wedge \beta=B \div \alpha, \text { or } \\
B \div \alpha \wedge \beta=B \div \beta, \text { or } \\
B \div \alpha \wedge \beta=B \div \alpha \cap B \div \beta\end{array}$ & $\begin{array}{l}\text { Assume conjunctive factoring } \\
\text { (the word "Either" is omitted } \\
\text { to save space) }\end{array}$ \\
\hline 3 & $\begin{array}{l}{[\delta \in B \div \alpha \wedge \beta \text { iff } \delta \in B \div \alpha], \text { or }} \\
{[\delta \in B \div \alpha \wedge \beta \text { iff } \delta \in B \div \beta], \text { or }} \\
{[\delta \in B \div \alpha \wedge \beta \text { iff } \delta \in B \div \alpha \text { and } \delta \in B \div \beta]}\end{array}$ & 2 (by the set theory) \\
\hline 4 & $\begin{array}{l}{[\delta \notin B \div \alpha \wedge \beta \text { iff } \delta \notin B \div \alpha], \text { or }} \\
{[\delta \notin B \div \alpha \wedge \beta \text { iff } \delta \notin B \div \beta], \text { or }} \\
{[\delta \notin B \div \alpha \wedge \beta \text { iff } \delta \notin B \div \alpha \text { or } \delta \notin B \div \beta]}\end{array}$ & $\begin{array}{l}3 \text { (negating both sides of each } \\
\text { iff statement) }\end{array}$ \\
\hline 5 & $\begin{array}{c}{[[\delta \in B \text { and } \delta \notin B \div \alpha \wedge \beta] \text { iff }} \\
[\delta \in B \text { and } \delta \notin B \div \alpha]], \text { or } \\
{[[\delta \in B \text { and } \delta \notin B \div \alpha \wedge \beta] \text { iff }} \\
[\delta \in B \text { and } \delta \notin B \div \beta]], \text { or } \\
{[[\delta \in B \text { and } \delta \notin B \div \alpha \wedge \beta] \text { iff }} \\
{[\delta \in B \text { and } \delta \notin B \div \alpha] \text { or }} \\
[\delta \in B \text { and } \delta \notin B \div \beta]]\end{array}$ & $\begin{array}{l}4 \text { (adding the conjunct } \\
\delta \in B \text { to both sides } \\
\text { of each iff statement) }\end{array}$ \\
\hline 6 & 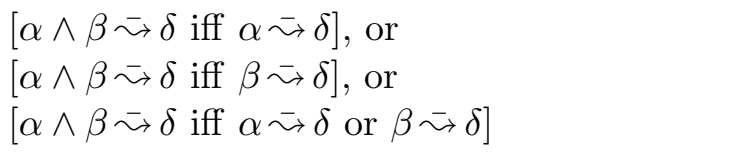 & 1,$5 ; \mathrm{CCDF}^{B}$ derived \\
\hline
\end{tabular}

Theorem 42 (Main Characterization). Let the relations $\leadsto$ and $\div$ for belief base $B$ be such that $\div$ satisfies inclusion, $B \div \alpha \subseteq B$, and that Cond $\leadsto$ holds. Then, $\div$ is a saturated kernel contraction that satisfies conjunctive factoring if and only if $\leadsto$ is a strong base dependence that satisfies $\mathrm{CCDF}^{B}$.

Proof. This axiomatic characterization theorem proof closely resembles the proof of the Characterization Theorem 34 (§A.4). The only difference is that here for the right to left direction, we need to use Theorem 40, and for the left to right direction, we use Theorem 41. Everything else stays the same, and thus is omitted here for brevity. 


\section{A.7 Proofs for $\S 5$ : Strong Base Dependence as a Reversible Generalization of Dependence}

Theorem 43 (Dependence Generalization). Let relations $\leadsto, \sim$ and $\div$ for belief base $B$ be such that Cond $\sim$ and Cond $\leadsto$ hold respectively and inclusion is satisfied. In the case where $B$ is logically closed, $B=\mathrm{Cn}(B)$,

(1) the following are logically equivalent:

a) $\leadsto$ is a strong base dependence

b) $\leadsto$ is a dependence that satisfies Def- $K$, Cond-ID, Disj, $\mathrm{LE}^{l}, \mathrm{LE}^{r}, \mathrm{CCl}^{r}$ and $\mathrm{CCD}_{0}^{r}$

c) $\div$ is a saturated kernel contraction

d) $\div$ is a basic AGM contraction function, which satisfies $(\div 1)-(\div 6)$

(2) if any one of 1.a-1.d above holds, then $\leadsto$ reduces to $\leadsto$ :

$\alpha \leadsto \beta$ iff $\alpha \leadsto \beta$.

Proof.

Part (1): We show 1.a-1.d are logically equivalent using s 6, 12 and 37.

\begin{tabular}{|c|c|c|}
\hline 1 & $B=\operatorname{Cn}(B)$ & Logical Closure \\
\hline 2 & $B \div \alpha \subseteq B$ & inclusion \\
\hline 3 & $\alpha \leadsto \beta \overline{\text { iff }} \beta \in \operatorname{Cn}(B)$ and $\beta \notin \operatorname{Cn}(B \div \alpha)$ & Cond $\leadsto$ \\
\hline 4 & $\alpha \leadsto \beta$ iff $\beta \in B$ and $\beta \notin B \div \alpha$ & Cond $\leadsto$ \\
\hline 5 & 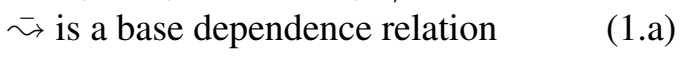 & Assumption \\
\hline 6 & $\div$ is a saturated kernel contraction & 2, 4, 5 and Theorem 37 \\
\hline 7 & $\begin{array}{l}\div \text { is a basic AGM contraction which } \\
\quad \text { satisfies }(\div 1)-(\div 6)\end{array}$ & 1,6 and Theorem 6 \\
\hline 8 & $\sim$ is a dependence relation & 1, 2, 3, 7 and Theorem 12 \\
\hline 9 & $\begin{array}{l}\text { Lines } 5 \text { through } 8 \text { (corresponding to } 1 . \mathrm{a}-1 . \mathrm{d} \text { ) } \\
\text { Theorems } 6,12 \text { and } 37 \text { connecting these } \mathrm{li}\end{array}$ & $\begin{array}{l}\text { are logically equivalent as all } \\
\text { es use logical equivalence }\end{array}$ \\
\hline \multicolumn{3}{|c|}{$\begin{array}{l}\text { Part (2): We start by assuming one of } 1 . \text { a-1.d holds, and by Part }(1) \text { it means } \\
\text { all of them hold. Thus, }(\div 1) \text { holds and by Theorem } 24 \leadsto \text { and } \leadsto \text { are equivalent. }\end{array}$} \\
\hline 10 & One of the lines 5 to 8 (or $1 . \mathrm{a}-1 . \mathrm{d}$ ) holds & Assumption for Part (2) \\
\hline 11 & Line 7 holds (so $(\div 1)-(\div 6)$ hold $)$ & 9,10 (because all lines 5 to 8 hold) \\
\hline 12 & $\div$ satisfies $(\div 1)$ (a.k.a. closure) & 11 \\
\hline 13 & $\alpha \leadsto \beta$ iff $\alpha \leadsto \beta$ & $1,3,4,12$ and Theorem 24 \\
\hline
\end{tabular}

Lemma 44. In the special case where base $B$ is logically closed, an operator $\div$ on $B$ is an $A G M$ contraction satisfying $(\div 1)-(\div 6),(\div 7)$ and $(\div 8)$ if and only if $\div$ is a saturated kernel contraction that satisfies conjunctive factoring. 


Proof.
\begin{tabular}{r|l|l}
\hline 1 & $B=\mathrm{Cn}(B)$ & Logical Closure \\
\hline 2 & Let $\div$ be a saturated kernel contraction & Assumption \\
3 & Let $\div$ also satisfy conjunctive factoring & Assumption \\
4 & $\div$ satisfies $(\div 1)-(\div 6)$ & 1,2 and Theorem 6 \\
5 & $\div$ satisfies $(\div 7)$ and $(\div 8)$ & $1,3,4$ and Theorem 1 \\
6 & $\div$ satisfies $(\div 1)-(\div 8)$ & 4,5 \\
\hline 7 & Let $\div$ satisfy $(\div 1)-(\div 6)$ & Assumption \\
8 & Let $\div$ also satisfy $(\div 7)$ and $(\div 8)$ & Assumption \\
9 & $\div$ is a saturated kernel contraction & 1,7 and Theorem 6 \\
10 & $\div$ satisfies conjunctive factoring & $1,7,8$ and Theorem 1 \\
\hline 11 & $\div$ satisfies $(\div 1)-(\div 8)$ if and only if & $2-6,7-10$ \\
& $\quad \div$ is a saturated kernel contraction that & \\
& also satisfies conjunctive factoring & \\
\hline
\end{tabular}

Theorem 45 (Dependence Generalization with Conjunction). Let relations $\leadsto, \leadsto$ and $\div$ for belief base $B$ be such that Cond $\leadsto$ and Cond $\sim$ hold respectively and inclusion is satisfied. In the case where $B$ is logically closed, $B=\mathrm{Cn}(B)$,

(1) the following are logically equivalent:

a) $\lesssim$ is a strong base dependence that satisfies $\mathrm{CCDF}^{B}$

b) $\leadsto$ is a dependence, which satisfies Def- $K$, Cond-ID, Disj, $\mathrm{LE}^{l}, \mathrm{LE}^{r}, \mathrm{CCl}^{l}, \mathrm{CCl}^{r}, \mathrm{CCD}_{0}^{l}$ and $\mathrm{CCD}_{0}^{r}$

c) $\div$ is a saturated kernel contraction that satisfies conjunctive factoring

d) $\div$ is an AGM contraction function, which satisfies $(\div 1)-(\div 6),(\div 7)$ and $(\div 8)$

(2) if any one of 1.a-1.d above holds, then $\leadsto$ reduces to $\leadsto$ :

$$
\alpha \leadsto \beta \text { iff } \alpha \leadsto \beta \text {. }
$$

Proof (theorem originally on page 122).

Part (1): We show 1.a-1.d are logically equivalent using Theorems 11, 42 and 44.

\begin{tabular}{|c|c|c|}
\hline 1 & $B=\operatorname{Cn}(B)$ & Logical Closure \\
\hline 2 & $B \div \alpha \subseteq B$ & inclusion \\
\hline 3 & $\alpha \leadsto \beta$ iff $\beta \in \mathrm{Cn}(B)$ and $\beta \notin \mathrm{Cn}(B \div \alpha)$ & Cond $\leadsto$ \\
\hline 4 & $\alpha \leadsto \beta$ iff $\beta \in B$ and $\beta \notin B \div \alpha$ & Cond $\leadsto$ \\
\hline 5 & $\begin{array}{l}\leadsto \text { is a base dependence relation that } \\
\text { also satisfies } C^{-} C D F^{B}\end{array}$ & Assumption \\
\hline 6 & $\begin{array}{l}\div \text { is a saturated kernel contraction that } \\
\text { also satisfies conjunctive factoring (1.c) }\end{array}$ & 2, 4, 5 and Theorem 42 \\
\hline 7 & $\begin{aligned} \div & \text { is an AGM contraction which } \\
& \text { satisfies }(\div 1)-(\div 8)\end{aligned}$ & 1, 6 and Lemma 44 \\
\hline 8 & $\leadsto$ is a dependence relation & 1, 2, 3, 7 and Theorem 11 \\
\hline 9 & $\begin{array}{l}\text { Lines } 5 \text { through } 8 \text { (corresponding to } 1 . a-1 . d \text { ) } \\
\text { Theorems } 11,42 \text { and } 44 \text { connecting these } 1\end{array}$ & $\begin{array}{l}\text { are logically equivalent as all } \\
\text { ines use logical equivalence }\end{array}$ \\
\hline
\end{tabular}


Part (2): We show : We start by assuming one of 1.a-1.d holds, and by Part (1) it means all of them hold. Thus, $(\div 1)$ holds and by Theorem $24 \leadsto$ and $\leadsto$ are equivalent.

\begin{tabular}{l|l|l} 
& One of the lines 5 to 8 (or 1. a-1.d) holds & Assumption for Part $(2)$ \\
11 & Line 7 holds (so $(\div 1)-(\div 8)$ hold) & 9,10 (because all lines 5 to 8 hold) \\
12 & $\div$ satisfies $(\div 1)$ (a.k.a. closure) & 11 \\
13 & $\alpha \leadsto \beta$ iff $\alpha \leadsto \beta$ & $1,3,4,12$ and Theorem 24 \\
\hline
\end{tabular}

\section{Acknowledgements}

We would like to thank the referees for their insightful comments and criticisms of the submitted version. James Delgrande gratefully acknowledges financial support from the Natural Sciences and Engineering Research Council of Canada.

\section{References}

Alchourrón, C. E., Gärdenfors, P., \& Makinson, D. (1985). On the logic of theory change: Partial meet contraction and revision functions. Journal of Symbolic Logic, 50, 510-530.

Chopra, S., \& Parikh, R. (2000). Relevance sensitive belief structures. Annals of Mathematics and Artificial Intelligence, 28(1), 259-285.

Cuenca Grau, B., Halaschek-Wiener, C., \& Kazakov, Y. (2007). History matters: Incremental ontology reasoning using modules.. pp. 183-196.

Delgrande, J. P., \& Pelletier, F. J. (1998). A formal analysis of relevance. Erkenntnis, 49(2), 137173.

Falappa, M., García, A., Kern-Isberner, G., \& Simari, G. (2011). Stratified belief bases revision with argumentative inference. Journal of Philosophical Logic, 1, 1-33.

Fariñas del Cerro, L., \& Herzig, A. (1996). Belief change and dependence. In Proceedings of the 6th conference on Theoretical aspects of rationality and knowledge (TARK'96), pp. 147-161. Morgan Kaufmann Publishers Inc. San Francisco, CA, USA.

Fermé, E. (2001). Five faces of recovery. In Frontiers in belief revision, pp. 247-259. Springer.

Gärdenfors, P. (1978). On the logic of relevance. Synthese, 37(3), 351-367.

Gärdenfors, P. (1984). Epistemic importance and minimal changes of belief. Australasian Journal of Philosophy, 62, 136-157.

Gärdenfors, P. (1988). Knowledge in Flux: Modeling the Dynamics of Epistemic States. MIT Press, Cambridge, Mass.

Gärdenfors, P. (1990). Belief revision and relevance. In PSA: Proceedings of the Biennial Meeting of the Philosophy of Science Association, pp. 349-365. Philosophy of Science Association.

Gärdenfors, P. (2003). Belief Revision. Cambridge University. 
Gärdenfors, P., \& Makinson, D. (1988). Revisions of knowledge systems using epistemic entrenchment. In Proceedings of the 2nd conference on Theoretical aspects of reasoning about knowledge, pp. 83-95, Pacific Grove, California. Morgan Kaufmann Publishers Inc.

Hansson, S. O. (1993). Theory contraction and base contraction unified. The Journal of Symbolic Logic, 58(2), 602.

Hansson, S. O. (1994). Kernel contraction. Journal of Symbolic Logic, 1, 845-859.

Hansson, S. O. (1999). A Textbook of Belief Dynamics: Theory Change and Database Updating. Kluwer Academic Publishers.

Hansson, S. O. (2003). A dyadic representation of belief. In Gärdenfors, P. (Ed.), Belief Revision, pp. 89-121. Cambridge University.

Hansson, S. O. (2011). Logic of belief revision. In Zalta, E. N. (Ed.), The Stanford Encyclopedia of Philosophy (Fall 2011 edition).

Hansson, S. O., \& Wassermann, R. (2002). Local change. Studia Logica, 70(1), 49-76.

Ismail, H., \& Kasrin, N. (2010). High-level perception as focused belief revision. In Proceeding of the 2010 conference on ECAI 2010: 19th European Conference on Artificial Intelligence, p. $1145-1146$.

Ji, Q., Qi, G., \& Haase, P. (2008). A relevance-based algorithm for finding justifications of DL entailments. Tech. rep., Technical report, University of Karlsruhe.

Keynes, J. M. (1921). A Treatise on Probability. Courier Dover Publications.

Kourousias, G., \& Makinson, D. (2007). Parallel interpolation, splitting, and relevance in belief change. Journal of symbolic logic, 72(3), 994-1002.

Makinson, D. (1987). On the status of the postulate of recovery in the logic of theory change. Journal of Philosophical Logic, 16(4), 383-394.

Makinson, D. (2007). Propositional relevance through letter-sharing: review and contribution. In Bonanno, G., Delgrande, J., Lang, J., \& Rott, H. (Eds.), Formal models of belief change in rational agents, pp. 1-13. Internationales Begegnungs und Forschungszentrum fur Informatik (IBFI), Dagstuhl, Germany.

Makinson, D., \& Kourousias, G. (2006). Respecting relevance in belief change. Análisis filosófico, 26(1), 53-61.

Oveisi, M. (2013). Belief Change and Base Dependence. Ph.D. thesis, Simon Fraser University, Canada.

Oveisi, M., Delgrande, J. P., Pelletier, F. J., \& Popowich, F. (2014). Belief change and base dependence. In 14th International Conference on Principles of Knowledge Representation and Reasoning (KR)., pp. 151-159. AAAI Press.

Oveisi, M., Delgrande, J. P., Popowich, F., \& Pelletier, F. J. (2015). Kernel contraction and base dependence: redundancy in the base resulting in different types of dependence. In Proceedings of the 24th International Conference on Artificial Intelligence (IJCAI)., pp. 3134-3140. AAAI Press.

Parikh, R. (1999). Beliefs, belief revision, and splitting languages. Logic, language and computation, 2, 266-278. 
Perrussel, L., Marchi, J., \& Zhang, D. (2011). Characterizing relevant belief revision operators. AI 2010: Advances in Artificial Intelligence, 1, 42-51.

Riani, J., \& Wassermann, R. (2004). Using relevance to speed up inference. some empirical results. In Advances in artificial intelligence: SBIA 2004, 17th Brazilian Symposium on Artificial Intelligence, São Luis, Maranhão, Brazil, September 29-October 1, 2004: proceedings, pp. 21-30.

Suntisrivaraporn, B., Qi, G., Ji, Q., \& Haase, P. (2008). A modularization-based approach to finding all justifications for OWL DL entailments. In Proceedings of the 3rd Asian Semantic Web Conference on The Semantic Web, pp. 1-15. Springer.

Tarski, A. (1956). Logic, Semantics, Metamathematics. Papers from 1923 to 1938. Oxford, Clarendon Press, 30-36.

Wassermann, R. (1999). Resource Bounded Belief Revision - Thesis. Ph.D. thesis.

Williams, M.-A. (1994). On the logic of theory base change. In Logics in Artificial Intelligence, pp. 86-105.

Wu, M., Zhang, D., \& Zhang, M. (2011). Language splitting and relevance-based belief change in horn logic. In Twenty-Fifth AAAI Conference on Artificial Intelligence, pp. 268-273. 